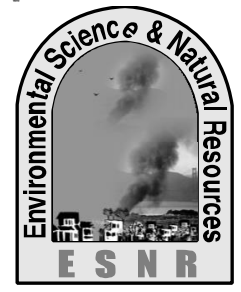

\title{
Analysis of Drought in the Northern Region of Bangladesh Using Standardized Precipitation Index (SPI)
}

\author{
R. Afrin", F. Hossain and S. A. Mamun \\ Department of Environmental Science and Resource Management, Mawlana Bhashani Science and \\ Technology University, Santosh, Tangail- 1902, Bangladesh \\ *Corresponding author: afrin.mbstu038@gmail.com
}

\begin{abstract}
Drought is an extended period when a region notes a deficiency in its water supply. The Standardized Precipitation Index (SPI) method was used in this study to analyze drought. Northern region of Bangladesh was the area of study. Monthly rainfall data of northern region of Bangladesh was obtained from the Meteorological Department of Bangladesh. Obtained rainfall data was from 1991 to 2011 and values from 2012 to 2026 were generated using Markov model. Then SPI values from 1991 to 2026 were calculated by using SPI formula for analyzing drought. Analysis with SPI method showed that droughts in northern region of Bangladesh varied from moderately dry to severely dry conditions and it may vary from moderately dry to severely dry conditions normally in future but in some cases extreme drought may also take place. From the study, it is observed that the northern region of Bangladesh has already experienced severe drought in 1991, 1992, 1994, 1995, 1997, 1998, 2000, 2003, 2005, 2007, 2009 and 2010. The region may experience severe drought in 2012, 2015, 2016, 2018, 2019, 2021, 2022, 2023, 2024, 2025 and 2026 and extreme drought in 2012, 2014, 2016, 2023 and 2024.
\end{abstract}

Key words: Drought, Markov model, Rainfall data

\section{Introduction}

A drought is an extended period when a region notes a deficiency in its water supply (Beran and Rodier, 1985). Generally, this occurs when a region receives consistently below average precipitation. It can have a substantial impact on the ecosystem and agriculture of the affected regions. Although droughts can persist for several years, even a short, intense drought can cause significant damage (Daniel, 2008). Many people in Bangladesh as well as the government perceive floods and cyclones as recurrent environmental hazards in the country. They also view that these two hazards are the main contributors to crop loss in the country. But in reality, droughts afflict the country at least as frequently as do major floods and cyclones, averaging about once in 2.5 years (Adnan, 1993; Ericksen, 1993; Hossain, 1990). Drought was the lone environmental factor to cause severe crop damage in Bangladesh in 1994. The northwestern region of the country, popularly known as North Bengal, experienced one of the most severe droughts of the century, which started in October 1994 and was broken in July 1995 with the onset of monsoon rain (Rahman, 1995). The continued drought in the northwestern districts of Bangladesh led to a shortfall of rice production of 3.5 million tons (Rahman and Biswas, 1995).

It is not possible to avoid drought but drought preparedness can be developed and drought impacts can be managed. The success of both depends, amongst others, on how well the droughts are defined and drought characteristics quantified (Smakhtin and Hughes, 2004). Droughts have often resulted in famine, displacement of people, homelessness, ill health, social disorder and in advance stages death (Odongkara, 2002). Most parts of Bangladesh depend on rain fed agriculture. But the amounts of rainfall and their duration time throughout the year are decreasing day by day. So drought has become a curse in many regions now-a-days. It is thus important to carry out drought studies so that when the disaster (drought) strikes, the occupants of the area are not caught unaware. Northern region of Bangladesh is taken as a case study area in this observation because it is considered as the most drought prone area of Bangladesh. The objective of the study is to generate future precipitation values using the Multi-period Markov model and analyze drought using the Standardized Precipitation Index (SPI). 


\section{Materials and Methods}

Study area
The Meteorological Department of Bangladesh has six stations in the northern part of Bangladesh and they were selected for the study (Table 1).

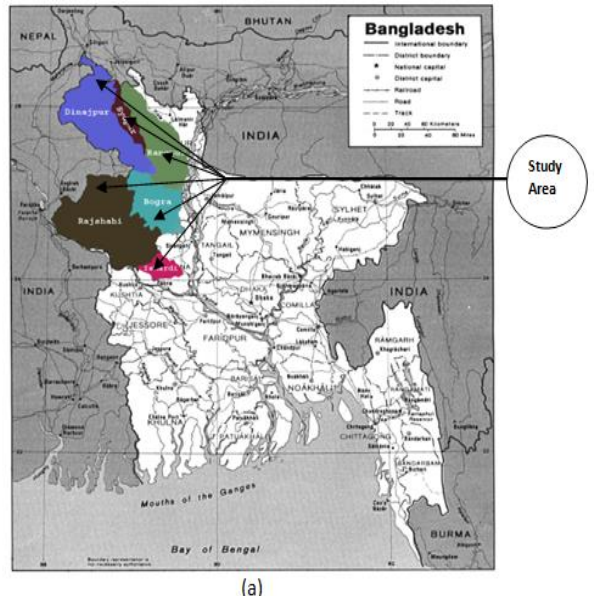

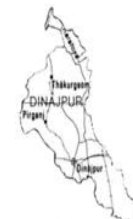

(b)

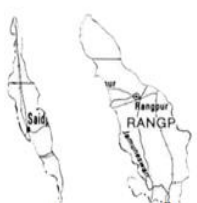

(c)

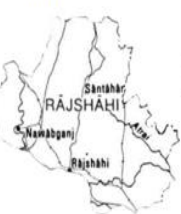

(e)

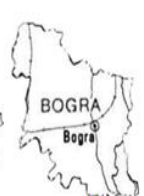

(f)

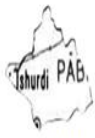

(g)

Fig. 1. Map showing the geographic position of the study area- (a) Bangladesh, (b) Dinajpur, (c) Sydpur, (d) Rangpur, (e) Rajshahi, (f) Bogra and (g) Ishurdi

Table 1. Names and locations of the selected stations

\begin{tabular}{|c|c|c|c|}
\hline Station No. & Name of the Station & Latitude & Longitude \\
\hline 1. & Dinajpur & $25^{\circ} 39^{\prime} \mathrm{N}$ & $88^{\circ} 41^{\prime} \mathrm{E}$ \\
\hline 2. & Rangpur & $25^{\circ} 44^{\prime} \mathrm{N}$ & $89^{\circ} 14^{\prime} \mathrm{E}$ \\
\hline 3. & Rajshahi & $24^{\circ} 22^{\prime} \mathrm{N}$ & $88^{\circ} 42^{\prime} \mathrm{E}$ \\
\hline 4. & Bogra & $24^{\circ} 51^{\prime} \mathrm{N}$ & $89^{\circ} 22^{\prime} \mathrm{E}$ \\
\hline 5. & Sydpur & $25^{\circ} 47^{\prime} \mathrm{N}$ & $88^{\circ} 53^{\prime} \mathrm{E}$ \\
\hline 6. & Ishurdi & $24^{\circ} 08^{\prime} \mathrm{N}$ & $89^{\circ} 03^{\prime} \mathrm{E}$ \\
\hline
\end{tabular}

\section{Data collection}

Monthly rainfall data records for the selected regions were obtained from the Meteorological Department, Agargaon, Dhaka.

\section{Generation of rainfall data}

Markov generation technique was used to generate and extend the rainfall data to the year 2042 for the northern region of Bangladesh. The formula is given below:

$$
=+(,-\quad)+1-
$$

where, $i$ is the periodic index (years),

$j$ is the annual index (months),
$Q_{i j}$ is the generated precipitation value (which will be set to zero, in case it is negative, but only after all the subsequent rainfall values have been generated),

is the mean precipitation in month $j$. It is given by:

$$
=\underline{\Sigma}
$$

where, $Q_{j}$ is the precipitation in month $j$ and $n$ is the number of years,

$b_{j}$ is a parameter given by<smiles>O=COc1ccccc1</smiles> 
$Q_{i-1, j-1}$ is the final recorded monthly precipitation from the preceding year or the generated value from the preceding year,

$\bar{Q}_{j-1}$ is the preceding mean monthly rainfall,

$t_{i}$ is a random number selected from a normal distribution having a zero mean and unit variance. The random number is given by $=R A N D($ in Microsoft Excel. Using $N O R M I N V(R A N D(), 0,1)$ in Microsoft Excel generates $t_{i}$. $\sigma_{j}$ is the standard deviation of observed precipitation values for the month $\mathrm{j}$ given by:

$\sigma_{j}=\sqrt{\frac{n \Sigma Q_{j}^{2}-\left(\Sigma Q_{j}\right)^{2}}{n(n-1)}}$

$r_{j}$ is the correlation coefficient for the relation of values in the period $j+1$ and period $j$. It is given by: $r_{j}=\frac{\operatorname{cov}\left(Q_{j}, Q_{j+1}\right)}{\sigma_{j} \cdot \sigma_{j+1}}$ where,

$\operatorname{cov}\left(Q_{j}, Q_{j+1}\right)=\frac{1}{n} \sum_{m=1}^{n}\left(Q_{j_{m}}-\bar{Q}_{j}\right)\left(Q_{j+1_{m}}-\bar{Q}_{j+1}\right)$

\section{Analysis of drought}

Analysis in terms of rainfall: The Standardized Precipitation Index (SPI)

The Standardized Precipitation Index (SPI) is a way of measuring drought and the SPI value is defined as the ratio of the difference between the measured rainfall and the long term mean to the standard deviation for any month (Table 2).

$S P I=\frac{Q_{j}-Q_{j}}{\sigma_{j}}$ where, $\sigma_{j}$ is the standard deviation for the month, $Q j$ and are the measured monthly and long term mean monthly rainfall respectively.

Table 2: The SPI scales

\begin{tabular}{|c|c|}
\hline SPI values & Weather condition \\
\hline $2.0+$ & Extremely wet \\
\hline 1.5 to 1.99 & Very wet \\
\hline 1.0 to 1.49 & Moderately wet \\
\hline-.99 to .99 & Near normal \\
\hline-1.0 to -1.49 & Moderately dry \\
\hline-1.5 to -1.99 & Severely dry \\
\hline-2 and less & Extremely dry \\
\hline
\end{tabular}

\section{Results and Discussions}

\section{Analysis of the collected rainfall data (1991-2011)}

Station No. 1 (Dinajpur) had experienced the highest rainfall in 2005 and the lowest in 1994. June, July, August and September were considered as the wettest periods having the highest rainfall amount over the study period. January, February, March, November and December were regarded as the driest periods having the lowest rainfall amount. April, May and October had moderate rainfall amount over the study periods (SI Table 1). 
SI Table 1. Monthly rainfall data records (mm) for Station No. 1 (Dinajpur) from 1991-2011

\begin{tabular}{|c|c|c|c|c|c|c|c|c|c|c|c|c|}
\hline Year & Jan & Feb & Mar & Apr & May & June & July & Aug & Sep & Oct & Nov & Dec \\
\hline 1991 & 27 & 4 & 1 & 18 & 400 & 376 & 184 & 282 & 647 & 29 & 0 & 44 \\
\hline 1992 & 6 & 6 & 1 & 5 & 87 & 212 & 535 & 228 & 483 & 76 & 6 & 4 \\
\hline 1993 & 42 & 0 & 29 & 82 & 308 & 495 & 218 & 565 & 361 & 64 & 15 & 0 \\
\hline 1994 & 23 & 22 & 0 & 50 & 146 & 294 & 148 & 122 & 248 & 89 & 0 & 0 \\
\hline 1995 & 3 & 12 & 10 & 0 & 54 & 287 & 472 & 593 & 1026 & 77 & 74 & 5 \\
\hline 1996 & 16 & 4 & 0 & 29 & 76 & 320 & 428 & 353 & 744 & 75 & 0 & 0 \\
\hline 1997 & 21 & 11 & 3 & 64 & 90 & 501 & 461 & 330 & 289 & 3 & 0 & 36 \\
\hline 1998 & 2 & 17 & 24 & 148 & 152 & 237 & 566 & 548 & 317 & 380 & 0 & 0 \\
\hline 1999 & 0 & 0 & 0 & 68 & 367 & 283 & 403 & 587 & 589 & 234 & 5 & 0 \\
\hline 2000 & 0 & 30 & 1 & 147 & 325 & 399 & 197 & 195 & 196 & 25 & 0 & 0 \\
\hline 2001 & 0 & 0 & 0 & 5 & 222 & 573 & 158 & 318 & 462 & 382 & 53 & 0 \\
\hline 2002 & 8 & 18 & 13 & 168 & 104 & 658 & 795 & 246 & 481 & 56 & 6 & 0 \\
\hline 2003 & 11 & 34 & 53 & 105 & 147 & 337 & 532 & 197 & 230 & 380 & 0 & 31 \\
\hline 2004 & 7 & 0 & 12 & 163 & 278 & 517 & 602 & 128 & 253 & 330 & 0 & 3 \\
\hline 2005 & 9 & 15 & 37 & 89 & 255 & 474 & 507 & 597 & 222 & 770 & 0 & 0 \\
\hline 2006 & 0 & 0 & 1 & 67 & 259 & 222 & 218 & 126 & 340 & 21 & 23 & 8 \\
\hline 2007 & 0 & 30 & 2 & 33 & 121 & 474 & 401 & 233 & 234 & 51 & 0 & 0 \\
\hline 2008 & 33 & 1 & 19 & 27 & 220 & 363 & 437 & 385 & 242 & 45 & 0 & 0 \\
\hline 2009 & 0 & 0 & 10 & 41 & 369 & 457 & 281 & 471 & 128 & 267 & 1 & 0 \\
\hline 2010 & 0 & 0 & 0 & 84 & 206 & 515 & 356 & 290 & 107 & 82 & 1 & 0 \\
\hline 2011 & 0 & 28 & 27 & 61 & 250 & 348 & 285 & 381 & 261 & 0 & 3 & 0 \\
\hline
\end{tabular}

Station No. 2 (Rangpur) had faced the highest rainfall in 2002 and the lowest rainfall in 1994. May, June, July, August and September had the highest rainfalls and
January, February, March, November and December had the lowest rainfalls over the study periods. April and October had moderate rainfalls (SI Table 2).

SI Table 2. Monthly rainfall data records (mm) for Station No. 2 (Rangpur) from 1991-2011

\begin{tabular}{|c|c|c|c|c|c|c|c|c|c|c|c|c|}
\hline Year & Jan & Feb & Mar & Apr & May & June & July & Aug & Sep & Oct & Nov & Dec \\
\hline 1991 & 38 & 1 & 1 & 41 & 302 & 593 & 162 & 264 & 726 & 106 & 2 & 27 \\
\hline 1992 & 13 & 10 & 0 & 89 & 245 & 348 & 248 & 367 & 552 & 129 & 4 & 2 \\
\hline 1993 & 49 & 0 & 30 & 62 & 190 & 633 & 499 & 572 & 358 & 91 & 26 & 0 \\
\hline 1994 & 16 & 29 & 28 & 49 & 212 & 427 & 202 & 97 & 135 & 106 & 0 & 0 \\
\hline 1995 & 3 & 3 & 4 & 1 & 158 & 369 & 568 & 388 & 804 & 51 & 109 & 3 \\
\hline 1996 & 13 & 0 & 0 & 39 & 232 & 396 & 479 & 353 & 333 & 159 & 0 & 0 \\
\hline 1997 & 19 & 14 & 3 & 113 & 230 & 276 & 523 & 338 & 410 & 16 & 2 & 27 \\
\hline 1998 & 0 & 12 & 50 & 173 & 202 & 333 & 473 & 458 & 255 & 409 & 0 & 0 \\
\hline 1999 & 0 & 0 & 7 & 228 & 336 & 447 & 436 & 829 & 337 & 303 & 8 & 0 \\
\hline 2000 & 1 & 15 & 3 & 283 & 416 & 438 & 166 & 232 & 172 & 19 & 0 & 0 \\
\hline 2001 & 0 & 1 & 23 & 38 & 235 & 481 & 341 & 346 & 550 & 470 & 7 & 0 \\
\hline 2002 & 8 & 6 & 38 & 376 & 290 & 913 & 582 & 287 & 521 & 99 & 7 & 0 \\
\hline 2003 & 8 & 23 & 109 & 143 & 160 & 573 & 633 & 183 & 206 & 340 & 0 & 24 \\
\hline 2004 & 9 & 0 & 39 & 196 & 347 & 352 & 653 & 133 & 464 & 484 & 0 & 3 \\
\hline 2005 & 11 & 9 & 61 & 93 & 271 & 428 & 671 & 400 & 328 & 581 & 0 & 0 \\
\hline 2006 & 0 & 1 & 2 & 85 & 367 & 472 & 271 & 96 & 235 & 120 & 8 & 25 \\
\hline 2007 & 0 & 47 & 16 & 39 & 233 & 568 & 558 & 149 & 277 & 138 & 12 & 0 \\
\hline 2008 & 36 & 1 & 49 & 74 & 273 & 444 & 232 & 396 & 227 & 175 & 0 & 0 \\
\hline 2009 & 0 & 0 & 9 & 158 & 270 & 336 & 304 & 832 & 77 & 231 & 0 & 0 \\
\hline 2010 & 0 & 0 & 0 & 169 & 237 & 650 & 346 & 240 & 332 & 122 & 4 & 2 \\
\hline 2011 & 0 & 20 & 13 & 28 & 261 & 306 & 389 & 542 & 366 & 6 & 1 & 0 \\
\hline
\end{tabular}


Station No. 3 (Rajshahi) had experienced the highest rainfalls in 1997 and the lowest in 1992. June, July, August, September and October were the wettest months over the study period. January, February, March,
November and December were considered as the driest months. April and May had moderate rainfalls (SI Table $3)$.

SI Table 3. Monthly rainfall data records (mm) for Station No. 3 (Rajshahi) from 1991-2011

\begin{tabular}{|c|c|c|c|c|c|c|c|c|c|c|c|c|}
\hline Year & Jan & Feb & Mar & Apr & May & June & July & Aug & Sep & Oct & Nov & Dec \\
\hline $\mathbf{1 9 9 1}$ & 6 & 0 & 19 & 25 & 157 & 211 & 286 & 96 & 484 & 122 & 0 & 92 \\
\hline $\mathbf{1 9 9 2}$ & 1 & 33 & 0 & 16 & 121 & 85 & 244 & 185 & 124 & 29 & 5 & 0 \\
\hline $\mathbf{1 9 9 3}$ & 0 & 5 & 55 & 70 & 65 & 477 & 247 & 177 & 316 & 157 & 54 & 0 \\
\hline $\mathbf{1 9 9 4}$ & 18 & 36 & 5 & 31 & 115 & 237 & 171 & 206 & 171 & 130 & 22 & 0 \\
\hline $\mathbf{1 9 9 5}$ & 17 & 31 & 9 & 8 & 91 & 291 & 287 & 270 & 370 & 13 & 44 & 1 \\
\hline $\mathbf{1 9 9 6}$ & 0 & 21 & 4 & 73 & 95 & 284 & 106 & 270 & 298 & 118 & 0 & 0 \\
\hline $\mathbf{1 9 9 7}$ & 8 & 35 & 19 & 56 & 53 & 242 & 763 & 468 & 348 & 4 & 44 & 22 \\
\hline $\mathbf{1 9 9 8}$ & 16 & 5 & 52 & 33 & 129 & 92 & 404 & 268 & 310 & 198 & 33 & 0 \\
\hline $\mathbf{1 9 9 9}$ & 0 & 0 & 0 & 9 & 144 & 348 & 349 & 354 & 502 & 155 & 1 & 0 \\
\hline $\mathbf{2 0 0 0}$ & 4 & 47 & 27 & 136 & 198 & 244 & 115 & 190 & 644 & 85 & 0 & 0 \\
\hline $\mathbf{2 0 0 1}$ & 0 & 0 & 9 & 13 & 209 & 324 & 338 & 209 & 95 & 184 & 1 & 0 \\
\hline $\mathbf{2 0 0 2}$ & 10 & 1 & 20 & 96 & 196 & 222 & 316 & 238 & 281 & 48 & 17 & 0 \\
\hline $\mathbf{2 0 0 3}$ & 3 & 18 & 64 & 45 & 84 & 280 & 230 & 128 & 262 & 292 & 0 & 6 \\
\hline $\mathbf{2 0 0 4}$ & 10 & 0 & 0 & 61 & 92 & 507 & 339 & 275 & 349 & 153 & 0 & 0 \\
\hline $\mathbf{2 0 0 5}$ & 14 & 1 & 104 & 27 & 108 & 92 & 492 & 161 & 131 & 275 & 0 & 0 \\
\hline $\mathbf{2 0 0 6}$ & 0 & 0 & 7 & 36 & 189 & 188 & 130 & 247 & 302 & 36 & 10 & 0 \\
\hline $\mathbf{2 0 0 7}$ & 0 & 27 & 59 & 13 & 260 & 313 & 364 & 236 & 309 & 76 & 1 & 0 \\
\hline $\mathbf{2 0 0 8}$ & 26 & 0 & 0 & 30 & 144 & 247 & 373 & 245 & 129 & 121 & 0 & 0 \\
\hline $\mathbf{2 0 0 9}$ & 1 & 7 & 28 & 0 & 131 & 126 & 183 & 240 & 282 & 45 & 0 & 0 \\
\hline $\mathbf{2 0 1 0}$ & 0 & 2 & 2 & 37 & 75 & 211 & 94 & 101 & 101 & 127 & 3 & 39 \\
\hline $\mathbf{2 0 1 1}$ & 6 & 0 & 10 & 94 & 187 & 341 & 144 & 454 & 203 & 35 & 1 & 0 \\
\hline
\end{tabular}

Station No. 4 (Bogra) had experienced the highest rainfalls in 1998 and the lowest rainfalls in 2006 over the last 21 years. May, June, July, August and September were the months having the highest rainfalls. January,
February, March, November and December had the lowest rainfalls over the study periods. April and October had moderate rainfalls (SI Table 4).

SI Table 4. Monthly rainfall data records (mm) for Station No. 4 (Bogra) from 1991-2011

\begin{tabular}{|c|c|c|c|c|c|c|c|c|c|c|c|c|}
\hline Year & Jan & Feb & Mar & Apr & May & June & July & Aug & Sep & Oct & Nov & Dec \\
\hline $\mathbf{1 9 9 1}$ & 4 & 14 & 8 & 48 & 416 & 240 & 404 & 207 & 733 & 105 & 0 & 112 \\
\hline $\mathbf{1 9 9 2}$ & 0 & 14 & 0 & 30 & 140 & 202 & 289 & 159 & 424 & 108 & 3 & 0 \\
\hline $\mathbf{1 9 9 3}$ & 65 & 8 & 14 & 58 & 268 & 466 & 432 & 266 & 240 & 95 & 2 & 0 \\
\hline $\mathbf{1 9 9 4}$ & 14 & 26 & 4 & 32 & 156 & 305 & 165 & 208 & 268 & 199 & 5 & 0 \\
\hline $\mathbf{1 9 9 5}$ & 16 & 18 & 14 & 0 & 111 & 462 & 524 & 438 & 477 & 117 & 70 & 1 \\
\hline $\mathbf{1 9 9 6}$ & 3 & 28 & 7 & 104 & 184 & 283 & 338 & 185 & 366 & 176 & 0 & 0 \\
\hline $\mathbf{1 9 9 7}$ & 10 & 11 & 9 & 140 & 102 & 199 & 298 & 245 & 220 & 29 & 2 & 27 \\
\hline $\mathbf{1 9 9 8}$ & 10 & 13 & 26 & 84 & 143 & 367 & 749 & 563 & 283 & 353 & 10 & 0 \\
\hline $\mathbf{1 9 9 9}$ & 0 & 0 & 0 & 9 & 261 & 283 & 343 & 397 & 145 & 133 & 17 & 0 \\
\hline $\mathbf{2 0 0 0}$ & 18 & 37 & 76 & 158 & 272 & 250 & 190 & 289 & 466 & 84 & 0 & 0 \\
\hline $\mathbf{2 0 0 1}$ & 0 & 0 & 0 & 22 & 223 & 299 & 185 & 115 & 344 & 203 & 8 & 0 \\
\hline $\mathbf{2 0 0 2}$ & 7 & 0 & 13 & 284 & 132 & 254 & 476 & 496 & 326 & 27 & 21 & 0 \\
\hline $\mathbf{2 0 0 3}$ & 4 & 53 & 72 & 113 & 214 & 364 & 219 & 220 & 156 & 264 & 0 & 13 \\
\hline $\mathbf{2 0 0 4}$ & 0 & 0 & 45 & 90 & 137 & 638 & 529 & 261 & 206 & 251 & 0 & 0 \\
\hline $\mathbf{2 0 0 5}$ & 5 & 9 & 58 & 72 & 138 & 130 & 471 & 328 & 356 & 523 & 0 & 1 \\
\hline $\mathbf{2 0 0 6}$ & 0 & 0 & 12 & 143 & 193 & 184 & 192 & 138 & 174 & 69 & 1 & 0 \\
\hline $\mathbf{2 0 0 7}$ & 0 & 18 & 25 & 28 & 92 & 732 & 320 & 256 & 302 & 131 & 15 & 0 \\
\hline $\mathbf{2 0 0 8}$ & 27 & 0 & 22 & 20 & 213 & 393 & 474 & 374 & 109 & 159 & 0 & 0 \\
\hline $\mathbf{2 0 0 9}$ & 0 & 3 & 3 & 49 & 205 & 128 & 194 & 570 & 169 & 89 & 0 & 0 \\
\hline $\mathbf{2 0 1 0}$ & 0 & 0 & 0 & 26 & 185 & 286 & 92 & 225 & 244 & 190 & 3 & 20 \\
\hline $\mathbf{2 0 1 1}$ & 1 & 0 & 7 & 145 & 194 & 193 & 175 & 606 & 389 & 0 & 11 & 0 \\
\hline
\end{tabular}


Station No. 5 (Sydpur) had experienced the highest rainfalls in 1999 and the lowest rainfalls in 1994. June, July, August and September were the months having the highest rainfalls. January, February, March, November and December had the lowest rainfalls. April, May and October had moderate rainfalls over the last 21 years (SI Table 5).

SI Table 5. Monthly rainfall data records (mm) for Station No. 5 (Sydpur) from 1991-2011

\begin{tabular}{|c|c|c|c|c|c|c|c|c|c|c|c|c|}
\hline Year & Jan & Feb & Mar & Apr & May & June & July & Aug & Sep & Oct & Nov & Dec \\
\hline $\mathbf{1 9 9 1}$ & 13 & 0 & 16 & 4 & 245 & 497 & 81 & 183 & 748 & 20 & 0 & 29 \\
\hline $\mathbf{1 9 9 2}$ & 16 & 5 & 0 & 0 & 133 & 219 & 318 & 317 & 498 & 74 & 2 & 12 \\
\hline $\mathbf{1 9 9 3}$ & 38 & 0 & 45 & 58 & 201 & 700 & 293 & 360 & 383 & 86 & 38 & 0 \\
\hline $\mathbf{1 9 9 4}$ & 19 & 40 & 21 & 49 & 143 & 224 & 176 & 75 & 255 & 242 & 0 & 0 \\
\hline $\mathbf{1 9 9 5}$ & 5 & 11 & 3 & 0 & 46 & 576 & 431 & 325 & 890 & 80 & 81 & 4 \\
\hline $\mathbf{1 9 9 6}$ & 18 & 0 & 0 & 10 & 195 & 259 & 501 & 227 & 468 & 101 & 0 & 0 \\
\hline $\mathbf{1 9 9 7}$ & 25 & 10 & 0 & 77 & 214 & 396 & 640 & 257 & 272 & 44 & 2 & 26 \\
\hline $\mathbf{1 9 9 8}$ & 0 & 10 & 23 & 148 & 217 & 322 & 725 & 838 & 472 & 381 & 0 & 3 \\
\hline $\mathbf{1 9 9 9}$ & 0 & 0 & 0 & 67 & 532 & 339 & 659 & 951 & 444 & 144 & 9 & 0 \\
\hline $\mathbf{2 0 0 0}$ & 0 & 1 & 2 & 219 & 303 & 368 & 138 & 206 & 201 & 27 & 1 & 0 \\
\hline $\mathbf{2 0 0 1}$ & 2 & 1 & 0 & 34 & 296 & 456 & 215 & 174 & 619 & 474 & 10 & 0 \\
\hline $\mathbf{2 0 0 2}$ & 11 & 5 & 162 & 380 & 137 & 755 & 865 & 215 & 307 & 27 & 6 & 0 \\
\hline $\mathbf{2 0 0 3}$ & 7 & 25 & 143 & 181 & 238 & 703 & 462 & 148 & 230 & 425 & 0 & 29 \\
\hline $\mathbf{2 0 0 4}$ & 4 & 0 & 21 & 138 & 435 & 546 & 735 & 253 & 340 & 313 & 1 & 1 \\
\hline $\mathbf{2 0 0 5}$ & 13 & 6 & 51 & 57 & 147 & 505 & 637 & 516 & 158 & 560 & 0 & 0 \\
\hline $\mathbf{2 0 0 6}$ & 0 & 0 & 2 & 90 & 633 & 525 & 211 & 74 & 404 & 76 & 20 & 18 \\
\hline $\mathbf{2 0 0 7}$ & 0 & 9 & 8 & 84 & 138 & 385 & 432 & 257 & 466 & 64 & 46 & 0 \\
\hline $\mathbf{2 0 0 8}$ & 32 & 1 & 19 & 29 & 253 & 572 & 453 & 408 & 128 & 44 & 0 & 0 \\
\hline $\mathbf{2 0 0 9}$ & 0 & 0 & 15 & 93 & 317 & 251 & 501 & 558 & 186 & 232 & 0 & 0 \\
\hline $\mathbf{2 0 1 0}$ & 0 & 0 & 0 & 131 & 202 & 642 & 288 & 263 & 259 & 163 & 2 & 1 \\
\hline $\mathbf{2 0 1 1}$ & 0 & 19 & 28 & 50 & 283 & 223 & 409 & 559 & 318 & 0 & 1 & 0 \\
\hline
\end{tabular}

Station No. 6 (Ishurdi) had faced the wettest periods in 1997 and the driest periods in 2010. May, June, July, August and September were the months having the highest rainfalls. January, February, March, November and December had the lowest rainfalls over the last 21 years. April and October were the months having moderate rain fall (SI Table 6).

SI Table 6. Monthly rainfall data records (mm) for Station No. 6 (Ishurdi) from 1991-2011

\begin{tabular}{|c|c|c|c|c|c|c|c|c|c|c|c|c|}
\hline Year & Jan & Feb & Mar & Apr & May & June & July & Aug & Sep & Oct & Nov & Dec \\
\hline $\mathbf{1 9 9 1}$ & 2 & 9 & 28 & 42 & 173 & 360 & 248 & 189 & 424 & 141 & 2 & 79 \\
\hline $\mathbf{1 9 9 2}$ & 0 & 40 & 0 & 18 & 132 & 73 & 252 & 207 & 295 & 38 & 5 & 3 \\
\hline $\mathbf{1 9 9 3}$ & 2 & 6 & 53 & 120 & 213 & 299 & 248 & 189 & 358 & 102 & 22 & 0 \\
\hline $\mathbf{1 9 9 4}$ & 24 & 45 & 3 & 31 & 155 & 336 & 111 & 150 & 99 & 82 & 2 & 0 \\
\hline $\mathbf{1 9 9 5}$ & 8 & 24 & 20 & 1 & 105 & 215 & 224 & 307 & 345 & 8 & 70 & 3 \\
\hline $\mathbf{1 9 9 6}$ & 0 & 25 & 17 & 104 & 81 & 295 & 155 & 271 & 269 & 183 & 0 & 0 \\
\hline $\mathbf{1 9 9 7}$ & 4 & 43 & 42 & 110 & 132 & 199 & 486 & 301 & 473 & 53 & 15 & 20 \\
\hline $\mathbf{1 9 9 8}$ & 16 & 12 & 68 & 109 & 269 & 101 & 413 & 225 & 285 & 96 & 42 & 0 \\
\hline $\mathbf{1 9 9 9}$ & 0 & 0 & 0 & 2 & 203 & 386 & 407 & 249 & 297 & 205 & 3 & 0 \\
\hline $\mathbf{2 0 0 0}$ & 8 & 61 & 20 & 206 & 233 & 212 & 241 & 147 & 551 & 129 & 0 & 0 \\
\hline $\mathbf{2 0 0 1}$ & 0 & 0 & 20 & 52 & 257 & 409 & 165 & 158 & 334 & 74 & 10 & 0 \\
\hline $\mathbf{2 0 0 2}$ & 15 & 2 & 62 & 72 & 177 & 296 & 253 & 227 & 240 & 75 & 47 & 0 \\
\hline $\mathbf{2 0 0 3}$ & 0 & 24 & 73 & 89 & 58 & 353 & 134 & 87 & 145 & 134 & 0 & 5 \\
\hline $\mathbf{2 0 0 4}$ & 0 & 0 & 0 & 127 & 125 & 292 & 397 & 156 & 541 & 151 & 0 & 0 \\
\hline $\mathbf{2 0 0 5}$ & 14 & 3 & 75 & 13 & 89 & 199 & 378 & 246 & 137 & 664 & 0 & 1 \\
\hline $\mathbf{2 0 0 6}$ & 0 & 0 & 2 & 74 & 213 & 199 & 233 & 244 & 277 & 4 & 40 & 0 \\
\hline $\mathbf{2 0 0 7}$ & 0 & 35 & 22 & 30 & 50 & 269 & 516 & 285 & 220 & 131 & 15 & 0 \\
\hline $\mathbf{2 0 0 8}$ & 37 & 2 & 12 & 35 & 145 & 206 & 295 & 208 & 256 & 108 & 0 & 0 \\
\hline $\mathbf{2 0 0 9}$ & 0 & 4 & 71 & 16 & 134 & 73 & 299 & 278 & 350 & 67 & 0 & 0 \\
\hline $\mathbf{2 0 1 0}$ & 0 & 3 & 0 & 46 & 100 & 162 & 175 & 127 & 109 & 105 & 3 & 63 \\
\hline $\mathbf{2 0 1 1}$ & 4 & 1 & 30 & 96 & 155 & 253 & 289 & 645 & 261 & 2 & 0 & 0 \\
\hline
\end{tabular}




\section{Generation of rainfall rata using Markov generation technique}

Each of the six stations had 21 years of record from 1991 to 2011. The Markov generation technique was used to generate data from 2012 to 2026, so that future stimulations could be made.

\section{Calculation of the required parameters}

In order to use the Markov generation equation in the generation of rainfall data, it was first necessary to calculate the required means, standard deviations and correlations for the given data on a monthly basis with reference to the period 1991 to 2011. Here, the parameters are calculated for Station no. 1 (Dinajpur) (Table 3) and then they are calculated in the same way for other stations.

Table 3. Calculated parameters for data generation for Station No. 1 (Dinajpur)

\begin{tabular}{|c|c|c|c|c|c|}
\hline Month, j & $\begin{array}{l}\text { Mean monthly precipitation, } \overline{\boldsymbol{Q}}_{\mathrm{j}} \\
(\mathbf{( m m )})\end{array}$ & $\begin{array}{c}\text { Standard deviation, } \\
\hat{O}_{\mathrm{j}}\end{array}$ & $\begin{array}{l}\text { Correlation } \\
\text { coefficient, } r_{j}\end{array}$ & $\mathbf{b}_{\mathbf{j}}$ & $\left(1-r_{j}{ }^{\wedge}\right)^{\wedge} 1 / 2$ \\
\hline Jan & 9.9 & 12.5 & -0.2 & -0.1 & 1 \\
\hline Feb & 11 & 11.9 & 0.4 & 0.4 & 0.9 \\
\hline Mar & 11.6 & $\begin{array}{ll}14.8 \\
\end{array}$ & 0.3 & 1.1 & 0.9 \\
\hline Apr & $\begin{array}{ll}69.2 \\
\end{array}$ & 52.2 & 0.1 & 0.2 & 1 \\
\hline May & 211.2 & 105.4 & 0.1 & 0.1 & 1 \\
\hline Jun & 397.2 & \begin{tabular}{ll|}
123.1 \\
\end{tabular} & 0.1 & 0.2 & 1 \\
\hline Jul & 389.7 & 171.1 & 0.1 & 0.1 & 1 \\
\hline Aug & 341.7 & 162 & 0.3 & 0.4 & 1 \\
\hline Sep & 374.3 & 225.1 & -0.2 & -0.2 & 1 \\
\hline Oct & 163.6 & 193.1 & -0.1 & -0.1 & 1 \\
\hline Nov & 8.9 & 19.3 & $\begin{array}{l}-0.1 \\
\end{array}$ & -0.1 & 1 \\
\hline Dec & 6.2 & 13.2 & 0.3 & 0.3 & 1 \\
\hline
\end{tabular}

\section{Generation of the required values}

Monthly rainfall values of 2011 were used as initial values for use in Equation 2 to generate values for the next year (2012). All calculations were done in Excel. Table 4 shows part of generation process with reference to the year 2013 for station no. 1 (Dinajpur). Then the generated rainfall values, covering the whole study period for the northern region of Bangladesh, have been attached in Table 5, 6, 7, 8, 9 and 10.

Table 4. Generation of synthetic monthly rainfall for Station No. 1 (Dinajpur)

\begin{tabular}{|c|c|c|c|c|c|c|c|c|c|c|c|}
\hline Year i & Monthj & $\overline{\mathbf{b}_{\mathbf{j}}}$ & $\begin{array}{l}\hat{O}_{j}^{* *(1-} \\
\left.\mathrm{r}_{\mathrm{j}}^{*} 2\right)^{\wedge} 1 / 2\end{array}$ & $\begin{array}{l}\bar{Q}_{\mathrm{j}} \\
(\mathrm{mm})\end{array}$ & $\begin{array}{l}\bar{Q}_{\mathrm{j}-1} \\
(\mathbf{m m})\end{array}$ & $\begin{array}{l}\text { Random } \\
\text { number }\end{array}$ & $\begin{array}{l}Q_{j-1, i-1} \\
(\mathbf{m m})\end{array}$ & $\overline{\mathbf{t}_{\mathrm{j}}}$ & $\begin{array}{l}\mathbf{b}_{\mathrm{j}}\left(\mathbf{Q}_{\mathrm{j}-1, \mathrm{i}-1^{-}}\right. \\
\left.\bar{Q}_{\mathrm{j}-1}\right)\end{array}$ & $\begin{array}{l}t_{j}{ }^{*} \hat{O}_{j}^{*} *(1- \\
\left.r_{j}^{\wedge} 2\right)^{\wedge} 1 / 2\end{array}$ & $\begin{array}{l}Q_{i, j} \\
(\mathbf{m m})\end{array}$ \\
\hline 2012 & 1 & -0.1 & 12.3 & 9.9 & 6.2 & 0.5 & 0 & 0.1 & 0.62 & 1.2 & 11.7 \\
\hline 2012 & 2 & 0.4 & 11.1 & 11 & 8.9 & 0.1 & 28 & -1.8 & 7.64 & -19.9 & -1.3 \\
\hline 2012 & 3 & 1.1 & 14 & 11.6 & 163.6 & 0.2 & 27 & -0.9 & -150.3 & -12.6 & -151.3 \\
\hline 2012 & 4 & 0.2 & 51.9 & $\begin{array}{ll}69.2 \\
\end{array}$ & 374.3 & 0.6 & 61 & 0.2 & -62.7 & 10.4 & 16.9 \\
\hline 2012 & 5 & 0.1 & 105 & 211.2 & 341.7 & 0.1 & 250 & -2 & -9.2 & -210 & -8 \\
\hline 2012 & 6 & 0.2 & 121.7 & 397.2 & 389.7 & 0.9 & 348 & 1.4 & -8.3 & 170.4 & 559.3 \\
\hline 2012 & 7 & 0.1 & 170.4 & 389.7 & 397.2 & 0.5 & 285 & 0.1 & -11.2 & 17 & 395.5 \\
\hline 2012 & 8 & 0.4 & 155 & 341.7 & 211.2 & 0.9 & 381 & 1.6 & 67.9 & 248 & 657.6 \\
\hline 2012 & 9 & -0.2 & 221.2 & 374.3 & 69.2 & 0.5 & 261 & -0.1 & -38.4 & -22.1 & 313.8 \\
\hline 2012 & 10 & -0.1 & 193.1 & 163.6 & 11.6 & 0.4 & 0 & -0.4 & 1.2 & -77.1 & 87.7 \\
\hline 2012 & 11 & -0.1 & 19.2 & 8.9 & 11 & 0.4 & 3 & -0.2 & 0.8 & -3.8 & 5.9 \\
\hline 2012 & 12 & 0.3 & 12.6 & 6.2 & 9.9 & 0.3 & 0 & -0.5 & -2.9 & -6.3 & -3.0 \\
\hline 2013 & 1 & -0.1 & 12.3 & 9.9 & 6.2 & 0.6 & 11.7 & 0.3 & $\begin{array}{l}-0.6 \\
\end{array}$ & 3.7 & 13 \\
\hline
\end{tabular}




\begin{tabular}{|c|c|c|c|c|c|c|c|c|c|c|c|}
\hline 2013 & 2 & 0.4 & 11.1 & 11 & 8.9 & 0.2 & -1.3 & -0.8 & -4.1 & -8.9 & -2 \\
\hline 2013 & 3 & 1.1 & 14 & 11.6 & 163.6 & 0.8 & -151.3 & 0.8 & -346.4 & 11.2 & -323.6 \\
\hline 2013 & 4 & 0.2 & 51.9 & 69.2 & 374.3 & 0.4 & 16.9 & -0.3 & -71.5 & -15.6 & -17.9 \\
\hline 2013 & 5 & 0.1 & 105 & 211.2 & 341.7 & 0.5 & -8 & -1.4 & -34.9 & -147 & 293 \\
\hline 2013 & 6 & 0.2 & 121.7 & 397.2 & 389.7 & 0.8 & 559.3 & 0.8 & 33.9 & 97.4 & 528.5 \\
\hline 2013 & 7 & 0.1 & 170.4 & 389.7 & 397.2 & 0.7 & 395.5 & 0.5 & -0.2 & 85.2 & 474.7 \\
\hline 2013 & 8 & 0.4 & 155 & 341.7 & 211.2 & 0.2 & 657.6 & -0.8 & 178.6 & -124 & 396.3 \\
\hline 2013 & 9 & -0.2 & 221.2 & 374.3 & 69.2 & 0.4 & 313.8 & -0.3 & -48.9 & -66.4 & 259 \\
\hline 2013 & 10 & -0.1 & 193.1 & 163.6 & 11.6 & 0.9 & 87.7 & 1.3 & -7.6 & 251 & 407 \\
\hline 2013 & 11 & -0.1 & 19.2 & 8.9 & 11 & 0.6 & 5.9 & 0.3 & 0.5 & 5.8 & 15.2 \\
\hline 2013 & 12 & 0.3 & 12.6 & 6.2 & 9.9 & 0.1 & -3.0 & -1.3 & -3.9 & -16.4 & -14.1 \\
\hline
\end{tabular}

Table 5. Synthetically generated rainfall values (in $\mathrm{mm}$ ) for Station No. 1 (Dinajpur)

\begin{tabular}{|c|c|c|c|c|c|c|c|c|c|c|c|c|}
\hline Year & Jan & Feb & Mar & Apr & May & June & July & Aug & Sep & Oct & Nov & Dec \\
\hline $\mathbf{2 0 1 2}$ & 11.7 & 0 & 0 & 16.9 & 0 & 559.3 & 395.5 & 657.6 & 313.8 & 87.7 & 5.9 & 0 \\
\hline $\mathbf{2 0 1 3}$ & 1.3 & 0 & 0 & 0 & 293 & 528.5 & 474.7 & 396.3 & 259 & 407 & 15.2 & 0 \\
\hline $\mathbf{2 0 1 4}$ & 21.5 & 3.3 & 0 & 0 & 111.8 & 473.6 & 533.8 & 338.2 & 358.5 & 568.2 & 0 & 16.6 \\
\hline $\mathbf{2 0 1 5}$ & 0 & 2.1 & 0 & 0 & 219.7 & 401.8 & 267.0 & 532 & 626.1 & 455.5 & 0 & 28.4 \\
\hline $\mathbf{2 0 1 6}$ & 14.0 & 0 & 0 & 87.1 & 115 & 95.4 & 342.6 & 206.5 & 0 & 293 & 0 & 7.0 \\
\hline $\mathbf{2 0 1 7}$ & 7.9 & 0 & 0 & 53.3 & 199 & 435.7 & 401.3 & 355.3 & 605.4 & 560.3 & 46.0 & 1.6 \\
\hline $\mathbf{2 0 1 8}$ & 8.5 & 0 & 0 & 46.5 & 207.4 & 503.8 & 407.2 & 414.8 & 421.9 & 533.6 & 41.9 & 0 \\
\hline $\mathbf{2 0 1 9}$ & 8.4 & 0 & 0 & 45.2 & 208.3 & 517.4 & 407.7 & 438.6 & 458.6 & 536.2 & 42.3 & 0 \\
\hline $\mathbf{2 0 2 0}$ & 8.5 & 0 & 0 & 44.9 & 208.4 & 520.1 & 407.8 & 448.2 & 451.3 & 535 & 42.3 & 0 \\
\hline $\mathbf{2 0 2 1}$ & 5 & 0 & 0 & 29.2 & 229.4 & 411.1 & 254.4 & 576 & 607.6 & 458.8 & 0 & 23.2 \\
\hline $\mathbf{2 0 2 2}$ & 6.3 & 0.7 & 0 & 26.2 & 231.5 & 389.3 & 239.1 & 627.1 & 576.3 & 466.5 & 0 & 30.4 \\
\hline $\mathbf{2 0 2 3}$ & 6.2 & 1.1 & 0 & 25.5 & 231.7 & 384.0 & 237.6 & 647.6 & 582.6 & 465.7 & 0 & 32.5 \\
\hline $\mathbf{2 0 2 4}$ & 8.7 & 0 & 0 & 40 & 210.7 & 493.4 & 390.8 & 547.3 & 426.5 & 0 & 0 & 30.6 \\
\hline $\mathbf{2 0 2 5}$ & 8.4 & 0 & 0 & 43.9 & 208.6 & 515.3 & 406.1 & 507.1 & 457.7 & 0 & 8.5 & 30.1 \\
\hline $\mathbf{2 0 2 6}$ & 8.5 & 0 & 0 & 44.6 & 208.4 & 519.7 & 407.6 & 491.1 & 451.4 & 0 & 0 & 29.9 \\
\hline
\end{tabular}

Table 6. Synthetically generated rainfall values (in $\mathrm{mm}$ ) for Station No. 2 (Rangpur)

\begin{tabular}{|c|c|c|c|c|c|c|c|c|c|c|c|c|}
\hline Year & Jan & Feb & Mar & Apr & May & June & July & Aug & Sep & Oct & Nov & Dec \\
\hline $\mathbf{2 0 1 2}$ & 19.6 & 2.6 & 22.4 & 105 & 214.2 & 307 & 449 & 226.7 & 321.7 & 380.5 & 12.2 & 6.2 \\
\hline $\mathbf{2 0 1 3}$ & 17.6 & 0 & 25.3 & 129.1 & 214.2 & 307.1 & 449 & 258.2 & 326.1 & 343 & 11.1 & 8.1 \\
\hline $\mathbf{2 0 1 4}$ & 17.8 & 0 & 26.1 & 136.3 & 214.2 & 307.1 & 449 & 255.1 & 325.7 & 330.3 & 6.4 & 8.6 \\
\hline $\mathbf{2 0 1 5}$ & 17.8 & 0 & 26.4 & 138.5 & 214.2 & 307.1 & 449 & 255.4 & 325.7 & 331.6 & 6.9 & 8.8 \\
\hline $\mathbf{2 0 1 6}$ & 15 & 0 & 26.5 & 139.1 & 214.2 & 307.1 & 449 & 255.3 & 325.7 & 331.5 & 6.9 & 8.8 \\
\hline $\mathbf{2 0 1 7}$ & 15.3 & 0 & 26.5 & 139.3 & 214.2 & 307.1 & 449 & 255.4 & 325.7 & 331.5 & 6.9 & 8.8 \\
\hline $\mathbf{2 0 1 8}$ & 11.1 & 0 & 0 & 139.4 & 214.2 & 307.1 & 449 & 255.3 & 325.7 & 331.5 & 6.9 & 6.8 \\
\hline $\mathbf{2 0 1 9}$ & 11.5 & 0 & 0 & 180.4 & 227.4 & 410.6 & 432.5 & 337.2 & 251.9 & 380.8 & 0 & 7.2 \\
\hline $\mathbf{2 0 2 0}$ & 11.5 & 0 & 0 & 192.7 & 227.4 & 421 & 432.5 & 329 & 259 & 375.9 & 0 & 7.4 \\
\hline $\mathbf{2 0 2 1}$ & 11.5 & 0 & 0 & 196.4 & 227.4 & 422 & 432.5 & 329.9 & 258.6 & 376.3 & 0 & 7.4 \\
\hline $\mathbf{2 0 2 2}$ & 12.9 & 0 & 0 & 197.5 & 227.4 & 422.1 & 432.5 & 329.8 & 258.6 & 376.3 & 0 & 6.4 \\
\hline $\mathbf{2 0 2 3}$ & 12.7 & 0 & 0 & 197.8 & 227.4 & 422.1 & 432.5 & 329.8 & 258.6 & 376.3 & 0 & 6 \\
\hline $\mathbf{2 0 2 4}$ & 11.4 & 1 & 0 & 165.1 & 253.7 & 422.1 & 432.5 & 329.8 & 258.6 & 376.3 & 0 & 6 \\
\hline $\mathbf{2 0 2 5}$ & 12.9 & 4.7 & 0 & 179.9 & 240.5 & 481.3 & 465.5 & 288.8 & 314 & 179 & 6.4 & 5 \\
\hline $\mathbf{2 0 2 6}$ & 12.7 & 6.2 & 0 & 184.3 & 240.5 & 487.2 & 465.5 & 292.9 & 308.4 & 198.8 & 4.6 & 4.7 \\
\hline
\end{tabular}


Table 7. Synthetically generated rainfall values (in $\mathrm{mm}$ ) for Station No. 3 (Rajshahi)

\begin{tabular}{|c|c|c|c|c|c|c|c|c|c|c|c|c|}
\hline Year & Jan & Feb & Mar & Apr & May & June & July & Aug & Sep & Oct & Nov & Dec \\
\hline $\mathbf{2 0 1 2}$ & 6.7 & 14.4 & 34.2 & 29.6 & 141 & 246.3 & 268.4 & 292.1 & 271.6 & 107.6 & 12.9 & 6.6 \\
\hline $\mathbf{2 0 1 3}$ & 6.7 & 12.8 & 31.8 & 21.1 & 145.6 & 258.0 & 296.4 & 261.6 & 264.8 & 100.4 & 11.7 & 5.9 \\
\hline $\mathbf{2 0 1 4}$ & 6.7 & 12.8 & 32.6 & 20.2 & 145.7 & 256.8 & 302.0 & 255.5 & 265.5 & 101.1 & 11.8 & 5.0 \\
\hline $\mathbf{2 0 1 5}$ & 6.7 & 12.5 & 33.1 & 20.9 & 182.9 & 255.8 & 309.3 & 260.1 & 266.8 & 104.2 & 12.2 & 6.1 \\
\hline $\mathbf{2 0 1 6}$ & 6.7 & 10.3 & 33.3 & 14.3 & 179.1 & 249.3 & 298.4 & 257.2 & 268.1 & 104.7 & 11.6 & 7.2 \\
\hline $\mathbf{2 0 1 7}$ & 6.7 & 11.3 & 32.2 & 15.1 & 151.1 & 255.5 & 308.6 & 246.0 & 266.5 & 102.3 & 12.7 & 6.5 \\
\hline $\mathbf{2 0 1 8}$ & 6.7 & 10.4 & 33.1 & 15.8 & 151.7 & 251.5 & 301.4 & 256.3 & 272.3 & 105.7 & 12.1 & 7.4 \\
\hline $\mathbf{2 0 1 9}$ & 6.7 & 11.3 & 32.2 & 14.8 & 152.8 & 256.4 & 296.8 & 252.6 & 266.1 & 101.4 & 11.6 & 6.0 \\
\hline $\mathbf{2 0 2 0}$ & 6.7 & 10.4 & 31 & 13.4 & 150.5 & 252.5 & 302.1 & 257.6 & 273.8 & 105 & 12.5 & 7.0 \\
\hline $\mathbf{2 0 2 1}$ & 6.7 & 13.0 & 31.9 & 15.3 & 153.4 & 256.3 & 315.6 & 260.5 & 264.6 & 99 & 11.6 & 6.1 \\
\hline $\mathbf{2 0 2 2}$ & 6.7 & 12.9 & 32.3 & 17.3 & 148.2 & 253.7 & 302.8 & 255.3 & 272.5 & 105.2 & 11.5 & 7 \\
\hline $\mathbf{2 0 2 3}$ & 6.7 & 13.7 & 30.9 & 19.2 & 145.5 & 252.8 & 290.9 & 258.1 & 280.2 & 102.2 & 11.9 & 6.1 \\
\hline $\mathbf{2 0 2 4}$ & 6.7 & 13.8 & 29.3 & 17.3 & 153.4 & 260.7 & 273.0 & 266.4 & 269.6 & 105.7 & 11.6 & 7.2 \\
\hline $\mathbf{2 0 2 5}$ & 6.7 & 12.4 & 32.6 & 18.5 & 145.5 & 258.8 & 291.1 & 277.7 & 266.4 & 104.6 & 12.7 & 7.8 \\
\hline $\mathbf{2 0 2 6}$ & 6.7 & 11.6 & 33.1 & 21.4 & 149 & 260.1 & 300.0 & 276.1 & 280.8 & 102.3 & 11.4 & 6 \\
\hline
\end{tabular}

Table 8. Synthetically generated rainfall values (in $\mathrm{mm}$ ) for Station No. 4 (Bogra)

\begin{tabular}{|c|c|c|c|c|c|c|c|c|c|c|c|c|}
\hline Year & Jan & Feb & Mar & Apr & May & June & July & Aug & Sep & Oct & Nov & Dec \\
\hline $\mathbf{2 0 1 2}$ & 28.8 & 4.4 & 0 & 0 & 352.7 & 303.6 & 503.4 & 225.0 & 201.7 & 100.8 & 26.8 & 11.7 \\
\hline $\mathbf{2 0 1 3}$ & 31.6 & 7.9 & 0 & 0 & 289.3 & 336.7 & 634.7 & 264.1 & 220.5 & 90.8 & 23.7 & 10.5 \\
\hline $\mathbf{2 0 1 4}$ & 25 & 8.2 & 0 & 0 & 285.6 & 405.7 & 657.4 & 289.5 & 175.5 & 126.9 & 32 & 18 \\
\hline $\mathbf{2 0 1 5}$ & 34.2 & 10.9 & 0 & 0 & 243.5 & 367.4 & 636.6 & 287 & 179.0 & 123.3 & 30.4 & 17.3 \\
\hline $\mathbf{2 0 1 6}$ & 29.2 & 13.1 & 0 & 0 & 260.3 & 355.9 & 628.3 & 287.3 & 179.6 & 123.6 & 30.7 & 17.4 \\
\hline $\mathbf{2 0 1 7}$ & 28.7 & 14.8 & 0 & 0 & 253.6 & 352.4 & 624.0 & 287.2 & 179.6 & 123.6 & 30.6 & 17.4 \\
\hline $\mathbf{2 0 1 8}$ & 18.3 & 16.2 & 0 & 0 & 227.3 & 410.4 & 429 & 346 & 251.4 & 76.8 & 5.8 & 4.0 \\
\hline $\mathbf{2 0 1 9}$ & 17.2 & 17.3 & 0 & 0 & 237.8 & 427.8 & 351 & 340 & 244.2 & 81.4 & 10.8 & 6.3 \\
\hline $\mathbf{2 0 2 0}$ & 11.2 & 21.9 & 0 & 0 & 211.8 & 256 & 394.5 & 355.4 & 273.7 & 127.8 & 9.8 & 6.1 \\
\hline $\mathbf{2 0 2 1}$ & 10.6 & 25.6 & 0 & 0 & 222.2 & 204.5 & 411.9 & 353.9 & 270.7 & 123.2 & 9 & 6.1 \\
\hline $\mathbf{2 0 2 2}$ & 16.4 & 27.3 & 0 & 0 & 218.0 & 189 & 418.9 & 354.0 & 271.0 & 123.6 & 10.2 & 6.1 \\
\hline $\mathbf{2 0 2 3}$ & 11.1 & 31.2 & 0 & 0 & 190.7 & 228.6 & 481.4 & 309.9 & 242.3 & 100.2 & 11.5 & 6.1 \\
\hline $\mathbf{2 0 2 4}$ & 10.6 & 34.3 & 0 & 0 & 201.6 & 240.5 & 506.4 & 314.4 & 245.1 & 102.5 & 11.2 & 6.1 \\
\hline $\mathbf{2 0 2 5}$ & 13.5 & 34.3 & 0 & 0 & 219 & 273.6 & 471.6 & 446.2 & 187.4 & 137.4 & 9.7 & 3.6 \\
\hline $\mathbf{2 0 2 6}$ & 18.2 & 31.8 & 0 & 0 & 212.1 & 283.5 & 457.7 & 432.0 & 193.2 & 133.9 & 10 & 3.9 \\
\hline
\end{tabular}


Table 9. Synthetically generated rainfall values (in $\mathrm{mm}$ ) for Station No. 5 (Sydpur)

\begin{tabular}{|c|c|c|c|c|c|c|c|c|c|c|c|c|}
\hline Year & Jan & Feb & Mar & Apr & May & June & July & Aug & Sep & Oct & Nov & Dec \\
\hline $\mathbf{2 0 1 2}$ & 12 & 11.5 & 0 & 143 & 217.3 & 327.4 & 434.6 & 493.5 & 302.5 & 140.4 & 19.2 & 5.9 \\
\hline $\mathbf{2 0 1 3}$ & 16.6 & 4.8 & 0 & 143 & 223.8 & 337.8 & 447.4 & 500.0 & 304.0 & 126.4 & 17.4 & 6.4 \\
\hline $\mathbf{2 0 1 4}$ & 10.9 & 5.8 & 0 & 152.9 & 443.7 & 474.7 & 191.4 & 362.1 & 516.1 & 0 & 0 & 5.5 \\
\hline $\mathbf{2 0 1 5}$ & 12 & 0 & 0 & 143 & 201.2 & 352.6 & 325.8 & 513.2 & 282.7 & 145.5 & 19 & 6.4 \\
\hline $\mathbf{2 0 1 6}$ & 8.6 & 1.1 & 0 & 117.2 & 266.8 & 459.2 & 374.3 & 292.2 & 344.6 & 142.1 & 7.1 & 8.5 \\
\hline $\mathbf{2 0 1 7}$ & 10.9 & 2.5 & 0 & 152.9 & 260.3 & 469.8 & 398.6 & 314.3 & 338.4 & 142.5 & 8.3 & 8.8 \\
\hline $\mathbf{2 0 1 8}$ & 12 & 0 & 0 & 143 & 219.5 & 352.1 & 429.4 & 517.9 & 300.4 & 126.2 & 18.4 & 6.7 \\
\hline $\mathbf{2 0 1 9}$ & 8.6 & 0 & 0 & 117.2 & 264 & 459.1 & 426.1 & 291.7 & 342.8 & 144.1 & 7.2 & 8.6 \\
\hline $\mathbf{2 0 2 0}$ & 12 & 0 & 0 & 143.0 & 219.2 & 350 & 443.2 & 520.2 & 299 & 126 & 18.6 & 6.7 \\
\hline $\mathbf{2 0 2 1}$ & 8.6 & 0 & 0 & 117.2 & 264 & 458.9 & 433 & 291.5 & 342.9 & 144.1 & 7.2 & 8.6 \\
\hline $\mathbf{2 0 2 2}$ & 16.6 & 0 & 0 & 108.3 & 191.6 & 418.9 & 577.9 & 360.1 & 319.3 & 174.8 & 14.5 & 10.8 \\
\hline $\mathbf{2 0 2 3}$ & 12 & 0 & 0 & 143 & 226.4 & 346 & 519.1 & 513.4 & 302.3 & 122 & 17.8 & 6.9 \\
\hline $\mathbf{2 0 2 4}$ & 8.6 & 0 & 0 & 117.2 & 264.3 & 458.5 & 470 & 292.2 & 342.6 & 144.5 & 7.3 & 8.6 \\
\hline $\mathbf{2 0 2 5}$ & 10.9 & 0 & 0 & 143.0 & 219.1 & 350.9 & 465.1 & 520.2 & 300.0 & 125 & 18.5 & 6.7 \\
\hline $\mathbf{2 0 2 6}$ & 12 & 0 & 0 & 143.0 & 223.7 & 340.2 & 462.7 & 497.4 & 304.3 & 127.9 & 17.4 & 6.5 \\
\hline
\end{tabular}

Table 10. Synthetically generated rainfall values (in $\mathrm{mm}$ ) for Station No. 6 (Ishurdi)

\begin{tabular}{|c|c|c|c|c|c|c|c|c|c|c|c|c|}
\hline Year & Jan & Feb & Mar & Apr & May & June & July & Aug & Sep & Oct & Nov & Dec \\
\hline $\mathbf{2 0 1 2}$ & 8.4 & 11.7 & 30 & 15 & 118.1 & 195.9 & 305.3 & 334 & 219.5 & 120.3 & 20.2 & 11.0 \\
\hline $\mathbf{2 0 1 3}$ & 8.4 & 10.7 & 30 & 0 & 110.7 & 207.3 & 310.2 & 333 & 227.8 & 108.5 & 16.1 & 9.9 \\
\hline $\mathbf{2 0 1 4}$ & 5.4 & 23.8 & 19.2 & 0 & 127.8 & 271.4 & 300.9 & 220.5 & 252.8 & 111.6 & 12.1 & 14.3 \\
\hline $\mathbf{2 0 1 5}$ & 8.4 & 9.4 & 27.8 & 0 & 112.7 & 192.2 & 308.9 & 334 & 221.1 & 109.4 & 17.7 & 9.6 \\
\hline $\mathbf{2 0 1 6}$ & 7.4 & 23.9 & 0 & 0 & 208.7 & 283.9 & 160.2 & 258.3 & 374.0 & 88.7 & 5.1 & 8 \\
\hline $\mathbf{2 0 1 7}$ & 8.4 & 9.4 & 18.6 & 0 & 128.9 & 189.7 & 266.6 & 333.0 & 196.9 & 111.6 & 19.1 & 10.2 \\
\hline $\mathbf{2 0 1 8}$ & 5.4 & 23.9 & 16.9 & 0 & 131.5 & 274.9 & 287.8 & 220.5 & 258.0 & 111.3 & 11.5 & 14.2 \\
\hline $\mathbf{2 0 1 9}$ & 7.4 & 20.6 & 24.7 & 0 & 144.4 & 257.9 & 294.1 & 220.5 & 246.7 & 111.3 & 13.1 & 13.8 \\
\hline $\mathbf{2 0 2 0}$ & 8.4 & 9.8 & 28.9 & 0 & 116 & 194.9 & 306.8 & 334 & 222.3 & 109.4 & 17.5 & 9.7 \\
\hline $\mathbf{2 0 2 1}$ & 13.3 & 14.6 & 19 & 0 & 97.9 & 235.9 & 375.4 & 283.5 & 240.5 & 111.5 & 18.6 & 10.1 \\
\hline $\mathbf{2 0 2 2}$ & 8.4 & 10.4 & 27.8 & 0 & 106.7 & 199.3 & 331.2 & 333 & 223.6 & 109.4 & 16.4 & 10.0 \\
\hline $\mathbf{2 0 2 3}$ & 12.3 & 14.5 & 18.8 & 0 & 96.1 & 282.5 & 382.7 & 258.3 & 253.6 & 105.8 & 14 & 3.7 \\
\hline $\mathbf{2 0 2 4}$ & 7.4 & 14.1 & 27.8 & 0 & 106.3 & 190 & 333.4 & 333.0 & 221 & 110 & 17.4 & 10.7 \\
\hline $\mathbf{2 0 2 5}$ & 8.4 & 10.4 & 29.6 & 0 & 108.4 & 208.5 & 318.6 & 334 & 227.5 & 109.5 & 16.7 & 10 \\
\hline $\mathbf{2 0 2 6}$ & 5.4 & 23.8 & 19.1 & 0 & 127.4 & 271.2 & 303.4 & 220.5 & 252.8 & 111.5 & 12.0 & 14.3 \\
\hline
\end{tabular}


Analysis of Drought

Standardized Precipitation Index (SPI) method
In the SPI method, the long term mean and standard deviation over the study period for each station were first calculated (Table 11).

Table 11. Calculated Long-term Mean and Standard Deviation for Station No. 1 (1991-2011)

\begin{tabular}{|c|c|c|c|c|c|c|c|c|c|c|c|c|}
\hline Months & 气్ & $\begin{array}{l}00 \\
\text { DI }\end{array}$ & $\sum^{\tilde{z}}$ & 艺 & $\stackrel{\mathrm{\Sigma}}{\mathrm{j}}^{\mathrm{j}}$ & $\Xi$ & $\Xi$ & $\stackrel{\infty 00}{\underset{\chi}{2}}$ & ڤे & $\ddot{0}$ & z & Uू. \\
\hline Long term mean & aे & $\Xi$ & $\stackrel{6}{=}$ & ণִ & $\frac{\stackrel{N}{\Xi}}{\sim}$ & $\frac{N}{\frac{1}{n}}$ & 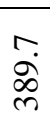 & $\underset{m}{\stackrel{\Xi}{f}}$ & $\frac{m}{\dot{N}}$ & $\begin{array}{l}0 \\
\text { ఠర }\end{array}$ & $\ddot{\infty}$ & ชู \\
\hline Standard deviation & $\stackrel{n}{\simeq}$ & $\stackrel{9}{=}$ & $\stackrel{\infty}{\stackrel{ \pm}{J}}$ & in & $\stackrel{⿱ 乛}{\stackrel{0}{0}}$ & $\overrightarrow{\stackrel{\Delta}{\beth}}$ & 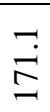 & $\underset{6}{6}$ & $\begin{array}{l}\overrightarrow{\vec{a}} \\
\vec{\Delta}\end{array}$ & $\vec{n}$ & $\stackrel{m}{=}$ & $\stackrel{\sim}{\ddot{n}}$ \\
\hline
\end{tabular}

Then the SPI values for each month in each year for each station over the whole study period were calculated (Table 12, 13, 14, 15, 16 and 17).

Table 12. Calculated SPI values for Station No. 1 (Dinajpur) (1991-2011)

\begin{tabular}{|c|c|c|c|c|c|c|c|c|c|c|c|c|}
\hline Year & Jan & Feb & Mar & Apr & May & Jun & Jul & Aug & Sep & Oct & Nov & Dec \\
\hline $\mathbf{1 9 9 1}$ & 1.4 & -0.6 & -0.7 & $-1^{*}$ & 1.8 & -0.2 & $-1.2^{*}$ & -0.4 & 1.2 & -0.7 & -0.5 & 2.9 \\
\hline $\mathbf{1 9 9 2}$ & -0.3 & -0.4 & -0.7 & $-1.2^{*}$ & $-1.2^{*}$ & $-1.5^{* *}$ & 0.9 & -0.7 & 0.5 & -0.5 & -0.2 & -0.2 \\
\hline $\mathbf{1 9 9 3}$ & 2.6 & -0.9 & 1.2 & 0.2 & 0.9 & 0.8 & $-1^{*}$ & 1.4 & -0.1 & -0.5 & 0.3 & -0.5 \\
\hline $\mathbf{1 9 9 4}$ & 1 & 0.9 & -0.8 & -0.4 & -0.6 & -0.8 & $-1.4^{*}$ & $-1.4^{*}$ & -0.6 & -0.4 & -0.5 & -0.5 \\
\hline $\mathbf{1 9 9 5}$ & -0.6 & 0.1 & -0.1 & $-1.3^{*}$ & $-1.5^{* *}$ & -0.9 & 0.5 & 1.6 & 2.9 & -0.5 & 3.4 & -0.1 \\
\hline $\mathbf{1 9 9 6}$ & 0.5 & -0.6 & -0.8 & -0.8 & $-1.3^{*}$ & -0.6 & 0.2 & 0.1 & 1.6 & -0.5 & -0.5 & -0.5 \\
\hline $\mathbf{1 9 9 7}$ & 0.9 & -0.1 & -0.6 & -0.1 & $-1.2^{*}$ & 0.8 & 0.4 & -0.1 & -0.4 & -0.8 & -0.5 & 2.3 \\
\hline $\mathbf{1 9 9 8}$ & -0.6 & 0.5 & 0.9 & 1.5 & -0.6 & $-1.3^{*}$ & 1 & 1.3 & -0.3 & 1.1 & -0.5 & -0.5 \\
\hline $\mathbf{1 9 9 9}$ & -0.8 & -0.9 & -0.8 & -0.1 & 1.5 & -0.9 & 0.1 & 1.5 & 1 & 0.4 & -0.2 & -0.5 \\
\hline $\mathbf{2 0 0 0}$ & -0.8 & 1.6 & -0.7 & 1.5 & 1.1 & 0 & $-1.1^{*}$ & -0.9 & -0.8 & -0.7 & -0.5 & -0.5 \\
\hline $\mathbf{2 0 0 1}$ & -0.8 & -0.9 & -0.8 & $-1.2^{*}$ & 0.1 & 1.4 & $-1.4 *$ & -0.2 & 0.4 & 1.1 & 2.3 & -0.5 \\
\hline $\mathbf{2 0 0 2}$ & -0.2 & 0.6 & 0.1 & 1.9 & $-1^{*}$ & 2.1 & 2.4 & -0.6 & 0.5 & -0.6 & -0.2 & -0.5 \\
\hline $\mathbf{2 0 0 3}$ & 0.1 & 1.9 & 2.8 & 0.7 & -0.6 & -0.5 & 0.8 & -0.9 & -0.6 & 1.1 & -0.5 & 1.9 \\
\hline $\mathbf{2 0 0 4}$ & -0.2 & -0.9 & 0 & 1.8 & 0.6 & 1 & 1.2 & $-1.3^{*}$ & -0.5 & 0.9 & -0.5 & -0.3 \\
\hline $\mathbf{2 0 0 5}$ & -0.1 & 0.3 & 1.7 & 0.4 & 0.4 & 0.6 & 0.7 & 1.6 & -0.7 & 3.1 & -0.5 & -0.5 \\
\hline $\mathbf{2 0 0 6}$ & -0.8 & -0.9 & -0.7 & -0.1 & 0.5 & $-1.4^{*}$ & $-1^{*}$ & $-1.3^{*}$ & -0.2 & -0.7 & 0.7 & 0.1 \\
\hline $\mathbf{2 0 0 7}$ & -0.8 & 1.6 & -0.7 & -0.7 & -0.9 & 0.6 & 0.1 & -0.7 & -0.6 & -0.6 & -0.5 & -0.5 \\
\hline $\mathbf{2 0 0 8}$ & 1.9 & -0.8 & 0.5 & -0.8 & 0.1 & -0.3 & 0.3 & 0.3 & -0.6 & -0.6 & -0.5 & -0.5 \\
\hline $\mathbf{2 0 0 9}$ & -0.8 & -0.9 & -0.1 & -0.5 & 1.5 & 0.5 & -0.6 & 0.8 & $-1.1 *$ & 0.5 & -0.4 & -0.5 \\
\hline $\mathbf{2 0 1 0}$ & -0.8 & -0.9 & -0.8 & 0.3 & -0.1 & 1 & -0.2 & -0.3 & $-1.2^{*}$ & -0.4 & -0.4 & -0.5 \\
\hline $\mathbf{2 0 1 1}$ & -0.8 & 1.4 & 1 & -0.2 & 0.4 & -0.4 & -0.6 & 0.2 & -0.5 & -0.9 & -0.3 & -0.5 \\
\hline
\end{tabular}

* Moderate drought, ** Severe drought, *** Extreme drought 
Table 13. Calculated SPI values for Station No. 2 (Rangpur) (1991-2011)

\begin{tabular}{|c|c|c|c|c|c|c|c|c|c|c|c|c|}
\hline Year & Jan & Feb & Mar & Apr & May & Jun & Jul & Aug & Sep & Oct & Nov & Dec \\
\hline $\mathbf{1 9 9 1}$ & 1.9 & -0.7 & -0.8 & -0.8 & 0.6 & 0.9 & $-1.5^{* *}$ & -0.5 & 2 & -0.6 & -0.3 & 2.1 \\
\hline $\mathbf{1 9 9 2}$ & 0.2 & 0.1 & -0.8 & -0.3 & -0.2 & -0.8 & $-1^{*}$ & 0.1 & 1 & -0.4 & -0.2 & -0.3 \\
\hline $\mathbf{1 9 9 3}$ & 2.7 & -0.7 & 0.3 & -0.6 & $-1.1^{*}$ & 1.1 & 0.5 & 1 & -0.1 & -0.6 & 0.7 & -0.5 \\
\hline $\mathbf{1 9 9 4}$ & 0.4 & 1.6 & 0.2 & -0.7 & -0.7 & -0.3 & $-1.3^{*}$ & $-1.3^{*}$ & $-1.2^{*}$ & -0.6 & -0.4 & -0.5 \\
\hline $\mathbf{1 9 9 5}$ & -0.5 & -0.5 & -0.7 & $*-1.2$ & $-1.6^{* *}$ & -0.7 & 0.9 & 0.2 & 2.4 & -0.9 & 4.2 & -0.2 \\
\hline $\mathbf{1 9 9 6}$ & 0.2 & -0.7 & -0.8 & -0.8 & -0.4 & -0.5 & 0.4 & -0.1 & -0.2 & -0.2 & -0.4 & -0.5 \\
\hline $\mathbf{1 9 9 7}$ & 0.6 & 0.4 & -0.7 & -0.1 & -0.5 & $-1.3^{*}$ & 0.7 & -0.1 & 0.2 & $-1.1^{*}$ & -0.3 & 2.1 \\
\hline $\mathbf{1 9 9 8}$ & -0.8 & 0.2 & 1 & 0.6 & -0.9 & -0.9 & 0.4 & 0.5 & -0.6 & 1.3 & -0.4 & -0.5 \\
\hline $\mathbf{1 9 9 9}$ & -0.8 & -0.7 & 0.6 & 1.2 & 1.2 & -0.1 & 0.1 & 2.3 & -0.2 & 0.6 & -0.1 & -0.5 \\
\hline $\mathbf{2 0 0 0}$ & -0.7 & 0.5 & -0.7 & 1.8 & 2.4 & -0.2 & $-1.5^{* *}$ & -0.6 & $-1^{*}$ & $-1.1^{*}$ & -0.4 & -0.5 \\
\hline $\mathbf{2 0 0 1}$ & -0.8 & -0.7 & -0.1 & -0.9 & -0.4 & 0.1 & -0.5 & -0.1 & 1 & 1.6 & -0.1 & -0.5 \\
\hline $\mathbf{2 0 0 2}$ & -0.2 & -0.3 & 0.5 & 2.7 & 0.5 & 3 & 1 & -0.3 & 0.8 & -0.6 & -0.1 & -0.5 \\
\hline $\mathbf{2 0 0 3}$ & -0.2 & 1.1 & 3.1 & 0.3 & $-1.5^{* *}$ & 0.7 & 1.3 & -0.9 & -0.9 & 0.9 & -0.4 & 1.8 \\
\hline $\mathbf{2 0 0 4}$ & -0.1 & -0.7 & 0.6 & 0.8 & 1.3 & -0.8 & 1.4 & $-1.1^{*}$ & 0.5 & 1.7 & -0.4 & -0.2 \\
\hline $\mathbf{2 0 0 5}$ & 0 & -0.1 & 1.4 & -0.3 & 0.2 & -0.3 & 1.6 & 0.2 & -0.2 & 2.3 & -0.4 & -0.5 \\
\hline $\mathbf{2 0 0 6}$ & -0.8 & -0.7 & -0.8 & -0.4 & 1.6 & 0 & -0.9 & $-1.3^{*}$ & -0.7 & -0.5 & -0.1 & 1.9 \\
\hline $\mathbf{2 0 0 7}$ & -0.8 & 3.1 & -0.3 & -0.8 & -0.4 & 0.7 & 0.9 & $-1^{*}$ & -0.5 & -0.4 & 0.4 & -0.5 \\
\hline $\mathbf{2 0 0 8}$ & 1.8 & -0.7 & 0.9 & -0.5 & 0.2 & -0.2 & $-1.1^{*}$ & 0.2 & -0.7 & -0.1 & -0.4 & -0.5 \\
\hline $\mathbf{2 0 0 9}$ & -0.8 & -0.7 & -0.5 & 0.4 & 0.2 & -0.9 & -0.7 & 2.3 & $-1.6^{* *}$ & 0.2 & -0.4 & -0.5 \\
\hline $\mathbf{2 0 1 0}$ & -0.8 & -0.7 & -0.8 & 0.5 & -0.4 & 1.2 & -0.4 & -0.6 & -0.2 & -0.5 & -0.2 & -0.3 \\
\hline $\mathbf{2 0 1 1}$ & -0.8 & 0.9 & -0.4 & -1 & 0 & $-1.1^{*}$ & -0.2 & 0.9 & 0 & $-1.1^{*}$ & -0.3 & -0.5 \\
\hline
\end{tabular}

* Moderate drought, ** Severe drought, *** Extreme drought

Table 14. Calculated SPI values for Station No. 3 (Rajshahi) (1991-2011)

\begin{tabular}{|l|c|c|c|c|l|l|l|l|l|l|l|l|}
\hline Year & Jan & Feb & Mar & Apr & May & Jun & July & Aug & Sep & Oct & Nov & Dec \\
\hline $\mathbf{1 9 9 1}$ & -0.1 & -0.8 & -0.2 & -0.5 & 0.4 & -0.4 & 0.0 & $-1.5^{* *}$ & 1.4 & 0.1 & -0.6 & 3.9 \\
\hline $\mathbf{1 9 9 2}$ & -0.7 & 1.3 & -0.8 & -0.8 & -0.3 & $-1.5^{* *}$ & -0.3 & -0.6 & $-1.2^{*}$ & $-1.1^{*}$ & -0.4 & -0.4 \\
\hline $\mathbf{1 9 9 3}$ & -0.9 & -0.5 & 1.1 & 0.8 & $-1.3^{*}$ & 2.0 & -0.2 & -0.6 & 0.2 & 0.5 & 2.4 & -0.4 \\
\hline $\mathbf{1 9 9 4}$ & 1.5 & 1.5 & -0.7 & -0.4 & -0.4 & -0.2 & -0.7 & -0.3 & -0.8 & 0.2 & 0.6 & -0.4 \\
\hline $\mathbf{1 9 9 5}$ & 1.4 & 1.2 & -0.5 & $-1^{*}$ & -0.8 & 0.3 & 0.0 & 0.3 & 0.6 & $-1.3^{*}$ & 1.9 & -0.3 \\
\hline $\mathbf{1 9 9 6}$ & -0.9 & 0.5 & -0.7 & 0.9 & -0.7 & 0.3 & $-1.2^{*}$ & 0.3 & 0.1 & 0.0 & -0.6 & -0.4 \\
\hline $\mathbf{1 9 9 7}$ & 0.2 & 1.4 & -0.2 & 0.4 & $-1.5^{* *}$ & -0.1 & 3.1 & 2.4 & 0.4 & $-1.4^{*}$ & 1.9 & 0.7 \\
\hline $\mathbf{1 9 9 8}$ & 1.2 & -0.5 & 1.0 & -0.3 & $-1.1^{*}$ & $-1.5^{* *}$ & 0.8 & 0.3 & 0.2 & 1.0 & 1.2 & -0.4 \\
\hline $\mathbf{1 9 9 9}$ & -0.9 & -0.8 & -0.8 & $-1^{*}$ & 0.2 & 0.8 & 0.4 & 1.2 & 1.5 & 0.5 & -0.6 & -0.4 \\
\hline $\mathbf{2 0 0 0}$ & -0.4 & 2.2 & 0.1 & 2.7 & 1.2 & -0.1 & $-1.1^{*}$ & -0.5 & 2.5 & -0.4 & -0.6 & -0.4 \\
\hline $\mathbf{2 0 0 1}$ & -0.9 & -0.8 & -0.5 & -0.9 & 1.4 & 0.6 & 0.3 & -0.3 & $-1.4^{*}$ & 0.9 & -0.6 & -0.4 \\
\hline $\mathbf{2 0 0 2}$ & 0.4 & -0.8 & -0.1 & 1.5 & 1.1 & -0.3 & 0.2 & -0.1 & -0.1 & -0.8 & 0.3 & -0.4 \\
\hline $\mathbf{2 0 0 3}$ & -0.5 & 0.3 & 1.5 & 0.1 & -0.9 & 0.2 & -0.4 & $-1.2^{*}$ & -0.2 & 2.2 & -0.6 & -0.1 \\
\hline $\mathbf{2 0 0 4}$ & 0.4 & -0.8 & -0.8 & 0.5 & -0.8 & 2.3 & 0.4 & 0.4 & 0.5 & 0.5 & -0.6 & -0.4 \\
\hline $\mathbf{2 0 0 5}$ & 0.1 & -0.8 & 2.9 & -0.5 & -0.5 & $-1.5^{* *}$ & 1.3 & -0.8 & $-1.1^{*}$ & 2.0 & -0.6 & -0.4 \\
\hline $\mathbf{2 0 0 6}$ & -0.9 & -0.8 & -0.6 & -0.2 & 1.0 & -0.6 & $-1^{*}$ & 0.1 & 0.1 & $-1^{*}$ & -0.1 & -0.4 \\
\hline $\mathbf{2 0 0 7}$ & -0.9 & 0.9 & 1.3 & -0.9 & 2.3 & 0.5 & 0.5 & -0.1 & 0.2 & -0.5 & -0.6 & -0.4 \\
\hline $\mathbf{2 0 0 8}$ & 2.5 & -0.8 & -0.8 & -0.4 & 0.2 & -0.1 & 0.6 & 0.1 & $-1.1^{*}$ & 0.1 & -0.6 & -0.4 \\
\hline $\mathbf{2 0 0 9}$ & -0.7 & -0.4 & 0.2 & $-1.3^{*}$ & -0.1 & $-1.2^{*}$ & -0.7 & 0.0 & -0.1 & -0.9 & -0.6 & -0.4 \\
\hline $\mathbf{2 0 1 0}$ & -0.9 & -0.7 & -0.8 & -0.2 & $-1.1^{*}$ & -0.4 & $-1.2^{*}$ & $-1.4^{*}$ & $-1.3^{*}$ & 0.2 & -0.5 & 1.5 \\
\hline $\mathbf{2 0 1 1}$ & -0.1 & -0.8 & -0.5 & 1.5 & 1.0 & 0.8 & -0.9 & 2.2 & -0.6 & 1.0 & -0.6 & -0.4 \\
\hline
\end{tabular}

* Moderate drought, ** Severe drought, *** Extreme drought 
Table 15. Calculated SPI values for Station No. 4 (Bogra) (1991-2011)

\begin{tabular}{|l|l|l|l|l|l|l|l|l|l|l|l|l|}
\hline Year & Jan & Feb & Mar & Apr & May & June & Jul & Aug & Sep & Oct & Nov & Dec \\
\hline $\mathbf{1 9 9 1}$ & -0.3 & 0.1 & -0.5 & -0.5 & 3.1 & -0.5 & 0.4 & -0.7 & 3.0 & -0.4 & -0.5 & 4.2 \\
\hline $\mathbf{1 9 9 2}$ & -0.6 & 0.1 & -0.8 & -0.7 & -0.7 & -0.7 & -0.3 & $-1^{*}$ & -0.8 & -0.4 & -0.3 & -0.3 \\
\hline $\mathbf{1 9 9 3}$ & 3.8 & -0.3 & -0.2 & -0.3 & 1.1 & 1.0 & 0.6 & -0.3 & -0.4 & -0.5 & -0.4 & -0.3 \\
\hline $\mathbf{1 9 9 4}$ & 0.4 & 1.0 & -0.7 & -0.7 & -0.5 & -0.1 & $-1.1^{*}$ & -0.7 & -0.3 & 0.3 & -0.2 & -0.3 \\
\hline $\mathbf{1 9 9 5}$ & 0.5 & 0.4 & -0.2 & $-1.2^{*}$ & $-1.1^{*}$ & 0.9 & 1.2 & 0.9 & 1.2 & -0.3 & 0.4 & -0.3 \\
\hline $\mathbf{1 9 9 6}$ & -0.4 & 1.1 & -0.5 & 0.4 & -0.1 & -0.2 & 0.0 & -0.9 & 0.4 & 0.2 & -0.5 & -0.3 \\
\hline $\mathbf{1 9 9 7}$ & 0.1 & -0.1 & -0.5 & 0.9 & $-1.2^{*}$ & -0.8 & -0.2 & -0.5 & -0.6 & $-1.1^{*}$ & -0.4 & 0.8 \\
\hline $\mathbf{1 9 9 8}$ & 0.1 & 0.1 & 0.3 & 0.1 & -0.6 & 0.3 & 2.5 & 1.7 & -0.1 & 1.6 & 0.1 & -0.3 \\
\hline $\mathbf{1 9 9 9}$ & -0.6 & -0.8 & -0.8 & $-1.0^{*}$ & 1.0 & -0.2 & 0.0 & 0.6 & $-1.1^{*}$ & -0.2 & 0.6 & -0.3 \\
\hline $\mathbf{2 0 0 0}$ & 0.6 & 1.7 & 2.4 & 1.2 & 1.1 & -0.4 & -0.9 & -0.2 & 1.1 & -0.6 & -0.5 & -0.3 \\
\hline $\mathbf{2 0 0 1}$ & -0.6 & -0.8 & -0.8 & -0.8 & 0.5 & -0.1 & -0.9 & $-1.3^{*}$ & 0.3 & 0.4 & 0.0 & -0.3 \\
\hline $\mathbf{2 0 0 2}$ & -0.1 & -0.8 & -0.3 & 3.0 & -0.8 & -0.4 & 0.9 & 1.2 & 0.1 & $-1.1^{*}$ & 0.8 & -0.3 \\
\hline $\mathbf{2 0 0 3}$ & -0.3 & 2.9 & 2.2 & 0.5 & 0.3 & 0.3 & -0.7 & -0.6 & $-1.0^{*}$ & 0.9 & -0.5 & 0.2 \\
\hline $\mathbf{2 0 0 4}$ & -0.6 & -0.8 & 1.1 & 0.2 & -0.7 & 2.1 & 1.2 & -0.3 & -0.7 & 0.8 & -0.5 & -0.3 \\
\hline $\mathbf{2 0 0 5}$ & -0.3 & -0.2 & 1.6 & -0.1 & -0.7 & $-1.2^{*}$ & 0.8 & 0.1 & 0.4 & 3.1 & -0.5 & -0.3 \\
\hline $\mathbf{2 0 0 6}$ & -0.6 & -0.8 & -0.3 & 0.9 & 0.0 & -0.9 & -0.9 & $-1.2^{*}$ & -0.9 & -0.7 & -0.4 & -0.3 \\
\hline $\mathbf{2 0 0 7}$ & -0.6 & 0.4 & 0.2 & -0.7 & $-1.3^{*}$ & 2.7 & -0.1 & -0.4 & -0.1 & -0.2 & 0.4 & -0.3 \\
\hline $\mathbf{2 0 0 8}$ & 1.2 & -0.8 & 0.1 & -0.9 & 0.3 & 0.5 & 0.8 & 0.4 & $-1.4^{*}$ & 0.0 & -0.5 & -0.3 \\
\hline $\mathbf{2 0 0 9}$ & -0.6 & -0.6 & -0.7 & -0.4 & 0.2 & $-1.2^{*}$ & -0.9 & 1.7 & -0.9 & -0.6 & -0.5 & -0.3 \\
\hline $\mathbf{2 0 1 0}$ & -0.6 & -0.8 & -0.8 & -0.8 & -0.1 & -0.2 & $-1.5^{* *}$ & -0.6 & -0.4 & 0.3 & -0.3 & 0.5 \\
\hline $\mathbf{2 0 1 1}$ & -0.5 & -0.8 & -0.5 & 1.0 & 0.1 & -0.8 & $-1^{*}$ & 2.0 & 0.6 & $-1.3^{*}$ & 0.2 & -0.3 \\
\hline
\end{tabular}

Table 16. Calculated SPI values for Station No. 5 (Sydpur) (1991-2011)

\begin{tabular}{|c|c|c|c|c|c|c|c|c|c|c|c|c|}
\hline Year & Jan & Feb & Mar & Apr & May & Jun & Jul & Aug & Sep & Oct & Nov & Dec \\
\hline $\mathbf{1 9 9 1}$ & 0.3 & -0.7 & -0.2 & $-1^{*}$ & -0.1 & 0.3 & $-1.6^{* *}$ & -0.7 & 1.9 & -0.9 & -0.5 & 2.2 \\
\hline $\mathbf{1 9 9 2}$ & 0.6 & -0.2 & -0.6 & $-1^{*}$ & -0.9 & $-1.4^{*}$ & -0.5 & -0.1 & 0.6 & -0.6 & -0.4 & 0.6 \\
\hline $\mathbf{1 9 9 3}$ & 2.5 & -0.7 & 0.4 & -0.4 & -0.4 & 1.5 & -0.7 & 0.1 & 0.0 & -0.5 & 1.3 & -0.6 \\
\hline $\mathbf{1 9 9 4}$ & 0.8 & 3.2 & -0.1 & -0.5 & -0.8 & $-1.3^{*}$ & $-1.2^{*}$ & $-1.2^{*}$ & -0.7 & 0.4 & -0.5 & -0.6 \\
\hline $\mathbf{1 9 9 5}$ & -0.4 & 0.4 & -0.5 & $-1^{*}$ & $-1.5^{* *}$ & 0.7 & -0.1 & -0.1 & 2.6 & -0.5 & 3.4 & -0.2 \\
\hline $\mathbf{1 9 9 6}$ & 0.7 & -0.7 & -0.6 & -0.9 & -0.4 & $-1.1^{*}$ & 0.3 & -0.5 & 0.4 & -0.4 & -0.5 & -0.6 \\
\hline $\mathbf{1 9 9 7}$ & 1.3 & 0.3 & -0.6 & -0.2 & -0.3 & -0.3 & 0.9 & -0.4 & -0.6 & -0.8 & -0.4 & 2.0 \\
\hline $\mathbf{1 9 9 8}$ & -0.8 & 0.3 & -0.1 & 0.6 & -0.3 & -0.8 & 1.3 & 2.2 & 0.5 & 1.3 & -0.5 & -0.3 \\
\hline $\mathbf{1 9 9 9}$ & -0.8 & -0.7 & -0.6 & -0.3 & 2.0 & -0.7 & 1.0 & 2.7 & 0.3 & -0.2 & -0.1 & -0.6 \\
\hline $\mathbf{2 0 0 0}$ & -0.8 & -0.6 & -0.6 & 1.4 & 0.4 & -0.5 & $-1.4^{*}$ & -0.6 & -0.9 & -0.9 & -0.5 & -0.6 \\
\hline $\mathbf{2 0 0 1}$ & -0.7 & -0.6 & -0.6 & -0.6 & 0.3 & 0.0 & $-1^{*}$ & -0.7 & 1.2 & 1.8 & -0.1 & -0.6 \\
\hline $\mathbf{2 0 0 2}$ & 0.1 & -0.2 & 3.0 & 3.2 & -0.8 & 1.8 & 2.0 & -0.5 & -0.4 & -0.9 & -0.2 & -0.6 \\
\hline $\mathbf{2 0 0 3}$ & -0.2 & 1.8 & 2.6 & 1.0 & -0.1 & 1.5 & 0.1 & -0.8 & -0.8 & 1.5 & -0.5 & 2.2 \\
\hline $\mathbf{2 0 0 4}$ & -0.5 & -0.7 & -0.1 & 0.5 & 1.3 & 0.6 & 1.4 & -0.4 & -0.2 & 0.9 & -0.5 & -0.5 \\
\hline $\mathbf{2 0 0 5}$ & 0.3 & -0.1 & 0.5 & -0.4 & -0.8 & 0.3 & 0.9 & 0.8 & $-1.2^{*}$ & 2.3 & -0.5 & -0.6 \\
\hline $\mathbf{2 0 0 6}$ & -0.8 & -0.7 & -0.6 & -0.1 & 2.7 & 0.4 & $-1^{*}$ & $-1.2^{*}$ & 0.1 & -0.6 & 0.5 & 1.2 \\
\hline $\mathbf{2 0 0 7}$ & -0.8 & 0.2 & -0.4 & -0.1 & -0.8 & -0.4 & -0.1 & -0.4 & -0.4 & -0.6 & 1.7 & -0.6 \\
\hline $\mathbf{2 0 0 8}$ & 1.9 & -0.6 & -0.2 & -0.7 & 0.0 & 0.7 & 0.1 & 0.3 & $-1.3^{*}$ & -0.8 & -0.5 & -0.5 \\
\hline $\mathbf{2 0 0 9}$ & -0.8 & -0.7 & -0.3 & 0.0 & 0.5 & $-1.2^{*}$ & 0.3 & 0.9 & $-1^{*}$ & 0.4 & -0.5 & -0.6 \\
\hline $\mathbf{2 0 1 0}$ & -0.8 & -0.7 & -0.6 & 0.5 & -0.4 & 1.1 & -0.7 & -0.3 & -0.6 & -0.1 & -0.4 & -0.5 \\
\hline $\mathbf{2 0 1 1}$ & 0.8 & 1.2 & 0.0 & -0.5 & 0.2 & $-1.3^{*}$ & -0.1 & 0.9 & -0.3 & $-1^{*}$ & -0.5 & -0.6 \\
\hline
\end{tabular}

* Moderate drought, ** Severe drought, *** Extreme drought 
Table 17. Calculated SPI values for Station No. 6 (Ishurdi) (1991-2011)

\begin{tabular}{|l|c|c|c|c|c|c|c|c|c|c|c|c|}
\hline Year & Jan & Feb & Mar & Apr & May & Jun & July & Aug & Sep & Oct & Nov & Dec \\
\hline $\mathbf{1 9 9 1}$ & -0.4 & -0.4 & -0.1 & -0.5 & 0.3 & 1.2 & -0.3 & -0.4 & 1.0 & 0.1 & -0.6 & 3.3 \\
\hline $\mathbf{1 9 9 2}$ & -0.6 & 1.3 & $-1.1^{*}$ & -0.9 & -0.3 & $-1.8^{* *}$ & -0.3 & -0.2 & -0.1 & -0.6 & -0.4 & -0.2 \\
\hline $\mathbf{1 9 9 3}$ & -0.4 & -0.5 & 0.9 & 1.0 & 1.0 & 0.5 & -0.3 & -0.4 & 0.5 & -0.1 & 0.4 & -0.4 \\
\hline $\mathbf{1 9 9 4}$ & 1.8 & 1.5 & $-1^{*}$ & -0.7 & 0.0 & 0.9 & $-1.5^{* *}$ & -0.7 & $-1.6^{* *}$ & -0.3 & -0.6 & -0.4 \\
\hline $\mathbf{1 9 9 5}$ & 0.2 & 0.4 & -0.3 & $-1.3^{*}$ & -0.8 & -0.3 & 2.0 & 0.7 & 0.4 & -0.8 & 2.9 & -0.2 \\
\hline $\mathbf{1 9 9 6}$ & -0.6 & 0.5 & -0.5 & 0.7 & $-1.1^{*}$ & 0.5 & $-1.1^{*}$ & 0.3 & -0.2 & 0.5 & -0.7 & -0.4 \\
\hline $\mathbf{1 9 9 7}$ & -0.2 & 1.4 & 0.5 & 0.8 & -0.3 & -0.5 & 1.8 & 0.6 & 1.4 & -0.5 & 0.1 & 0.5 \\
\hline $\mathbf{1 9 9 8}$ & 1.0 & -0.2 & 1.4 & 0.8 & 1.9 & $-1.5^{* *}$ & 3.7 & -0.1 & -0.1 & -0.2 & 1.4 & -0.4 \\
\hline $\mathbf{1 9 9 9}$ & -0.6 & -0.9 & $-1.1^{*}$ & $-1.2^{*}$ & 0.8 & 1.4 & 1.1 & 0.1 & -0.1 & 0.6 & -0.5 & -0.4 \\
\hline $\mathbf{2 0 0 0}$ & 0.2 & 2.4 & -0.3 & 2.7 & 1.3 & -0.4 & -0.4 & -0.8 & 2.0 & 0.1 & -0.7 & -0.4 \\
\hline $\mathbf{2 0 0 1}$ & -0.6 & -0.9 & -0.3 & -0.3 & 1.7 & 1.7 & $-1.0^{*}$ & -0.7 & 0.3 & -0.3 & -0.2 & -0.4 \\
\hline $\mathbf{2 0 0 2}$ & 0.9 & -0.8 & 1.2 & 0.1 & 0.4 & 0.5 & -0.3 & -0.1 & -0.5 & -0.3 & 1.7 & -0.4 \\
\hline $\mathbf{2 0 0 3}$ & -0.6 & 0.4 & 1.6 & 0.4 & $-1.5^{* *}$ & 1.1 & $-1.3^{*}$ & $-1.3^{*}$ & $-1.2^{*}$ & 0.1 & -0.7 & -0.2 \\
\hline $\mathbf{2 0 0 4}$ & -0.6 & -0.9 & $-1.1^{*}$ & 1.2 & -0.4 & 0.5 & 1.0 & -0.7 & 1.9 & 0.2 & -0.7 & -0.4 \\
\hline $\mathbf{2 0 0 5}$ & 0.8 & -0.7 & 1.7 & $-1.0^{*}$ & $-1^{*}$ & -0.5 & 0.8 & 0.1 & $-1.3^{*}$ & 4.0 & -0.7 & -0.3 \\
\hline $\mathbf{2 0 0 6}$ & -0.6 & -0.9 & -1.0 & 0.1 & 1.0 & -0.5 & 2.1 & 0.1 & -0.2 & -0.9 & 1.3 & -0.4 \\
\hline $\mathbf{2 0 0 7}$ & -0.6 & 1.0 & -0.3 & -0.7 & $-1.6^{* *}$ & 0.2 & 2.1 & 0.5 & -0.6 & 0.1 & 0.1 & -0.4 \\
\hline $\mathbf{2 0 0 8}$ & 3.1 & -0.8 & -0.6 & -0.6 & -0.1 & -0.4 & 0.1 & -0.2 & -0.3 & -0.1 & -0.7 & -0.4 \\
\hline $\mathbf{2 0 0 9}$ & -0.6 & -0.6 & 1.5 & $-1^{*}$ & -0.3 & $-1.8^{* *}$ & 0.2 & 0.4 & 0.4 & -0.4 & -0.7 & -0.4 \\
\hline $\mathbf{2 0 1 0}$ & -0.6 & -0.7 & $-1.1^{*}$ & -0.4 & -0.8 & -0.9 & -0.9 & -0.9 & $-1.5^{* *}$ & -0.1 & -0.5 & 2.5 \\
\hline $\mathbf{2 0 1 1}$ & -0.2 & -0.8 & 1.1 & 0.6 & 0.0 & 0.1 & 0.1 & 3.7 & -0.3 & -0.9 & -0.7 & -0.4 \\
\hline
\end{tabular}

* Moderate drought, ** Severe drought, *** Extreme drought

Future analysis of drought using SPI

For future analysis of drought, SPI values from 2012 to 2026 were calculated for the six stations and are shown in Table 18, $19,20,21,22$ and 23.

Table 18. Calculated SPI values for Station No. 1 (Dinajpur) (2012-2026)

\begin{tabular}{|c|c|c|c|c|c|c|c|c|c|c|c|c|}
\hline Year & Jan & Feb & Mar & Apr & May & June & July & Aug & Sep & Oct & Nov & Dec \\
\hline $\mathbf{2 0 1 2}$ & 0.5 & -0.3 & 0 & -0.7 & $-2.8^{* * *}$ & 1.0 & 0.3 & 1.4 & -0.8 & $-1.2^{*}$ & $-0.4^{*}$ & $-1.1^{*}$ \\
\hline $\mathbf{2 0 1 3}$ & 0.8 & -0.3 & 0 & $-1.4^{*}$ & 1.5 & 0.7 & 1.2 & -0.7 & $-1.1^{*}$ & 0.2 & 0.1 & $-1.1^{*}$ \\
\hline $\mathbf{2 0 1 4}$ & 2.6 & 3.0 & 0 & $-1.4^{*}$ & $-1.2^{*}$ & 0.2 & 1.9 & $-1.1^{*}$ & -0.5 & 1.0 & -0.7 & 0.1 \\
\hline $\mathbf{2 0 1 5}$ & $-1.9^{* *}$ & 1.8 & 0 & $-1.4^{*}$ & 0.4 & -0.4 & $-1.2^{*}$ & 0.4 & 1.1 & 0.4 & -0.7 & 0.9 \\
\hline $\mathbf{2 0 1 6}$ & 1.0 & -0.3 & 0 & 2.3 & $-1.1^{*}$ & $-3.1^{* * *}$ & -0.3 & $-2.2^{* * *}$ & $-2.7^{* * *}$ & -0.3 & -0.7 & -0.6 \\
\hline $\mathbf{2 0 1 7}$ & -0.3 & -0.3 & 0 & 0.8 & 0.1 & -0.1 & 0.3 & $-1^{*}$ & 1.0 & 0.9 & 1.7 & $-1^{*}$ \\
\hline $\mathbf{2 0 1 8}$ & -0.1 & -0.3 & 0 & 0.6 & 0.2 & 0.5 & 0.4 & -0.5 & -0.1 & 0.8 & 1.5 & $-1.1^{*}$ \\
\hline $\mathbf{2 0 1 9}$ & -0.1 & -0.3 & 0 & 0.5 & 0.2 & 0.6 & 0.4 & -0.3 & 0.1 & 0.8 & 1.5 & $-1.1^{*}$ \\
\hline $\mathbf{2 0 2 0}$ & -0.1 & -0.3 & 0 & 0.5 & 0.2 & 0.6 & 0.4 & -0.2 & 0.1 & 0.8 & 1.5 & $-1.1^{*}$ \\
\hline $\mathbf{2 0 2 1}$ & -0.9 & -0.3 & 0 & -0.2 & 0.5 & -0.3 & $-1.3 *$ & 0.8 & 1.0 & 0.5 & -0.7 & 0.5 \\
\hline $\mathbf{2 0 2 2}$ & -0.6 & 0.4 & 0 & -0.3 & 0.6 & -0.5 & $-1.5^{* *}$ & 1.2 & 0.8 & 0.5 & -0.7 & 1.1 \\
\hline $\mathbf{2 0 2 3}$ & -0.6 & 0.8 & 0 & -0.3 & 0.6 & -0.6 & $-1.5^{* *}$ & 1.3 & 0.9 & 0.5 & -0.7 & 1.2 \\
\hline $\mathbf{2 0 2 4}$ & -0.1 & -0.3 & 0 & 0.3 & 0.3 & 0.4 & 0.2 & 0.5 & -0.1 & $-1.6^{* *}$ & -0.7 & 1.1 \\
\hline $\mathbf{2 0 2 5}$ & -0.1 & -0.3 & 0 & 0.4 & 0.2 & 0.6 & 0.4 & 0.2 & 0.1 & $-1.6^{* *}$ & -0.3 & 1.0 \\
\hline $\mathbf{2 0 2 6}$ & -0.1 & -0.3 & 0 & 0.5 & 0.2 & 0.6 & 0.4 & 0.1 & 0.1 & $-1.6^{* *}$ & -0.7 & 1.0 \\
\hline
\end{tabular}

* Moderate drought, ** Severe drought, *** Extreme drought 
Table 19. Calculated SPI values for Station No. 2 (Rangpur) (2012-2026)

\begin{tabular}{|c|c|c|c|c|c|c|c|c|c|c|c|c|}
\hline Year & Jan & Feb & Mar & Apr & May & June & July & Aug & Sep & Oct & Nov & Dec \\
\hline 2012 & 1.9 & 0.8 & 0.9 & $-1.9 * *$ & -0.9 & $-1 *$ & 0.4 & $-1.6^{* *}$ & 0.8 & 0.7 & 1.8 & -0.6 \\
\hline 2013 & 1.2 & -0.5 & 1.2 & $-1.1 *$ & -0.9 & $-1 *$ & 0.4 & -0.8 & 0.9 & 0.1 & 1.5 & 0.7 \\
\hline 2014 & 1.3 & -0.5 & 1.2 & -0.8 & -0.9 & $-1 *$ & 0.4 & -0.9 & 0.9 & -0.1 & 0.4 & 1.1 \\
\hline 2015 & 1.3 & -0.5 & 1.2 & -0.8 & -0.9 & $-1^{*}$ & 0.4 & -0.9 & 0.9 & -0.1 & 0.6 & 1.3 \\
\hline 2016 & 0.3 & -0.5 & 1.3 & -0.7 & -0.9 & $-1 *$ & 0.4 & -0.9 & 0.9 & -0.1 & 0.6 & 1.3 \\
\hline 2017 & 0.4 & -0.5 & 1.3 & -0.7 & -0.9 & $-1^{*}$ & 0.4 & -0.9 & 0.9 & -0.1 & 0.6 & 1.3 \\
\hline 2018 & $-1.0^{*}$ & -0.5 & -0.8 & -0.7 & -0.9 & $-1 *$ & 0.4 & -0.9 & 0.9 & -0.1 & 0.6 & -0.2 \\
\hline 2019 & -0.9 & -0.5 & -0.8 & 0.6 & -0.9 & 0.5 & $-1 *$ & 1.3 & $-1.3 *$ & 0.7 & $-1.1 *$ & 0.1 \\
\hline 2020 & -0.9 & -0.5 & -0.8 & 1 & -0.9 & 0.6 & $-1 *$ & 1 & $-1.1 *$ & 0.7 & $-1.1 *$ & 0.2 \\
\hline 2021 & -0.9 & -0.5 & -0.8 & 1.2 & 0.2 & 0.7 & $-1 *$ & 1.1 & $-1.1 *$ & 0.7 & $-1.1 *$ & 0.2 \\
\hline 2022 & -0.4 & -0.5 & -0.8 & 1.2 & 0.2 & 0.7 & $-1 *$ & 1.1 & $-1.1 *$ & 0.7 & $-1.1 *$ & -0.5 \\
\hline 2023 & -0.5 & -0.5 & -0.8 & 1.2 & 0.2 & 0.7 & $-1 *$ & 1.1 & $-1.1 *$ & 0.7 & $-1.1 *$ & -0.8 \\
\hline 2024 & -0.9 & 0 & -0.8 & 0.1 & 2.3 & 0.7 & $-1 *$ & 1.1 & $-1.1 *$ & 0.7 & $-1.1 *$ & -0.8 \\
\hline 2025 & -0.4 & 1.9 & -0.8 & 0.6 & 1.3 & 1.5 & 1.8 & 0 & 0.5 & $-2.5^{*} * *$ & 0.4 & $-1.5^{* *}$ \\
\hline 2026 & -0.5 & 2.7 & -0.8 & 0.8 & 1.3 & 1.6 & 1.8 & 0.1 & 0.4 & $-2.2 * * *$ & 0 & $-1.7 * *$ \\
\hline
\end{tabular}

* Moderate drought, ** Severe drought, *** Extreme drought

Table 20. Calculated SPI values for Station No. 3 (Rajshahi) (2012-2026)

\begin{tabular}{|c|c|c|c|c|c|c|c|c|c|c|c|c|}
\hline Year & Jan & Feb & Mar & Apr & May & June & July & Aug & Sep & Oct & Nov & Dec \\
\hline 2012 & 3.6 & 1.7 & 1.7 & 2.8 & $-1^{*}$ & $-2.2 * * *$ & 4.5 & 2.6 & 0.3 & 1.8 & 1.8 & 0.1 \\
\hline 2013 & -0.3 & 0.4 & -0.4 & 0.7 & -0.6 & 0.8 & 6.7 & -0.1 & $-1 *$ & $-1.3^{*}$ & -0.6 & -0.9 \\
\hline 2014 & -0.3 & 0.4 & 0.3 & 0.5 & -0.6 & 0.5 & 7.1 & -0.6 & -0.9 & $-1^{*}$ & -0.4 & $-2.1 * * *$ \\
\hline 2015 & -0.3 & 0.2 & 0.7 & 0.6 & 2.5 & 0.2 & 7.7 & -0.2 & -0.6 & 0.3 & 0.4 & -0.6 \\
\hline 2016 & -0.3 & $-1.5 * *$ & 0.9 & $-1^{*}$ & 2.2 & $-1.4 *$ & 6.9 & -0.4 & -0.4 & 0.5 & -0.8 & 0.9 \\
\hline 2017 & -0.3 & -0.7 & -0.1 & 0.8 & -0.2 & 0.1 & 7.7 & $-1.4^{*}$ & -0.7 & -0.5 & 1.4 & -0.1 \\
\hline 2018 & -0.3 & $-1.4 *$ & 0.7 & -0.6 & -0.1 & -0.9 & 7.1 & -0.5 & 0.4 & 1 & 0.2 & 1.2 \\
\hline 2019 & -0.3 & -0.7 & -0.1 & -0.8 & -0.1 & 0.4 & 6.7 & -0.8 & -0.7 & -0.9 & -0.8 & -0.7 \\
\hline 2020 & -0.3 & $-1.4 *$ & $-1^{*}$ & $-1.2 *$ & -0.2 & -0.6 & 7.1 & -0.4 & 0.7 & 0.7 & 1 & 0.6 \\
\hline 2021 & -0.3 & 0.6 & -0.3 & -0.7 & 0 & 0.3 & 8.2 & -0.1 & $-1 *$ & $-1.9 * *$ & -0.8 & -0.6 \\
\hline 2022 & -0.3 & 0.5 & 0.1 & -0.2 & -0.4 & -0.3 & 7.2 & -0.6 & 0.5 & 0.7 & $-1^{*}$ & 0.6 \\
\hline 2023 & -0.3 & 1.1 & $-1.1 *$ & 0.2 & -0.6 & -0.5 & 6.3 & -0.4 & 2 & -0.5 & -0.2 & -0.6 \\
\hline 2024 & -0.3 & 1.2 & $-2.5 * * *$ & -0.2 & 0 & 1.4 & 4.8 & 0.4 & -0.1 & 1 & -0.8 & 0.9 \\
\hline 2025 & -0.3 & 0.1 & 0.3 & 0.1 & -0.6 & 1 & 6.3 & 1.3 & -0.7 & 0.5 & 1.4 & 1.7 \\
\hline 2026 & -0.3 & -0.5 & 0.7 & 0.8 & -0.3 & 1.3 & 6.9 & 1.2 & 2.1 & -0.5 & $-1.2 *$ & -0.7 \\
\hline
\end{tabular}

* Moderate drought, ** Severe drought, *** Extreme drought 
Table 21. Calculated SPI values for Station No. 4 (Bogra) (2012-2026)

\begin{tabular}{|c|c|c|c|c|c|c|c|c|c|c|c|c|}
\hline Year & Jan & Feb & Mar & Apr & May & June & July & Aug & Sep & Oct & Nov & Dec \\
\hline 2012 & 1 & $-1.5 * *$ & 0 & 0 & 2.6 & -0.1 & -0.1 & $-1.7 * *$ & -0.5 & -0.6 & 0.9 & 0.4 \\
\hline 2013 & 1.3 & $-1.2 *$ & 0 & 0 & 1.1 & 0.4 & 0.3 & $-1.0^{*}$ & -0.1 & $-1.2 *$ & 0.6 & 0.2 \\
\hline 2014 & 0.6 & $-1.1^{*}$ & 0 & 0 & 1 & 1.3 & 0.4 & -0.6 & $-1.2 *$ & 0.7 & 1.4 & 1.6 \\
\hline 2015 & 1.6 & -0.9 & 0 & 0 & 0 & 0.8 & 0.3 & -0.7 & $-1.1 *$ & 0.5 & 1.3 & 1.4 \\
\hline 2016 & 1.1 & -0.7 & 0 & 0 & 0.4 & 0.6 & 0.3 & -0.7 & $-1.1 *$ & 0.5 & 1.3 & 1.5 \\
\hline 2017 & 1 & -0.5 & 0 & 0 & 0.3 & 0.6 & 0.3 & 4.9 & $-1.1 *$ & 0.5 & 1.3 & 1.5 \\
\hline 2018 & -0.2 & -0.4 & 0 & 0 & -0.3 & 1.3 & -0.2 & 0.3 & 0.8 & $-1.9 * *$ & $-1.2 *$ & $-1 *$ \\
\hline 2019 & -0.4 & -0.3 & 0 & 0 & -0.1 & 1.5 & -0.4 & 0.2 & 0.6 & $-1.6^{* *}$ & -0.7 & -0.6 \\
\hline 2020 & $-1.1 *$ & 0.2 & 0 & 0 & -0.7 & -0.7 & -0.3 & 0.5 & 1.4 & 0.8 & -0.8 & -0.6 \\
\hline 2021 & $-1.1 *$ & 0.6 & 0 & 0 & -0.5 & $-1.4^{*}$ & -0.2 & 0.5 & 1.3 & 0.5 & -0.8 & -0.6 \\
\hline 2022 & -0.5 & 0.7 & 0 & 0 & -0.6 & $-1.6^{* *}$ & -0.2 & 0.5 & 1.3 & 0.5 & -0.7 & -0.6 \\
\hline 2023 & $-1.1 *$ & 1.1 & 0 & 0 & $-1.2 *$ & $-1.0 *$ & -0.1 & -0.3 & 0.6 & -0.7 & -0.6 & -0.6 \\
\hline 2024 & $-1.1 *$ & 1.4 & 0 & 0 & $-1 *$ & -0.9 & -0.1 & -0.2 & 0.6 & -0.5 & -0.6 & -0.6 \\
\hline 2025 & -0.8 & 1.4 & 0 & 0 & -0.5 & -0.5 & -0.1 & 2 & -0.9 & 1.3 & -0.8 & $-1 *$ \\
\hline 2026 & -0.2 & 1.2 & 0 & 0 & -0.7 & -0.3 & -0.1 & 1.8 & -0.7 & 1.1 & -0.7 & $-1 *$ \\
\hline
\end{tabular}

* Moderate drought, ** Severe drought, *** Extreme drought

Table 22. Calculated SPI values for Station No. 5 (Sydpur) (2012-2026)

\begin{tabular}{|c|c|c|c|c|c|c|c|c|c|c|c|c|}
\hline Year & Jan & Feb & Mar & Apr & May & June & July & Aug & Sep & Oct & Nov & Dec \\
\hline 2012 & 0.2 & 3 & 0 & 0.5 & -0.5 & $-1.2 *$ & 0.1 & 0.7 & -0.5 & 0.3 & 1 & $-1 *$ \\
\hline 2013 & 2 & 0.9 & 0 & 0.5 & -0.4 & $-1^{*}$ & 0.2 & 0.8 & -0.5 & -0.1 & 0.7 & -0.7 \\
\hline 2014 & -0.2 & 1.2 & 0 & 1.2 & 3.3 & 1.3 & $-2.7 * * *$ & -0.5 & 3.4 & 3.4 & $-2.1 * * *$ & $-1.3^{*}$ \\
\hline 2015 & 0.2 & -0.5 & 0 & 0.5 & -0.8 & -0.7 & $-1.2 *$ & 0.9 & -0.8 & 0.4 & 0.9 & -0.7 \\
\hline 2016 & $-1.1 *$ & -0.2 & 0 & $-1.2 *$ & 0.3 & 1.0 & -0.6 & $-1.2 *$ & 0.3 & 0.4 & $-1 *$ & 0.7 \\
\hline 2017 & -0.2 & 0.2 & 0 & 1.2 & 0.2 & 1.2 & -0.3 & $-1^{*}$ & 0.2 & 0.4 & -0.8 & 0.9 \\
\hline 2018 & 0.2 & -0.5 & 0 & 0.5 & -0.5 & -0.8 & 0.0 & 1 & -0.5 & -0.1 & 0.8 & -0.5 \\
\hline 2019 & $-1.1 *$ & -0.5 & 0 & $-1.2 *$ & 0.3 & 1.0 & -0.1 & $-1.2 *$ & 0.2 & 0.4 & $-1 *$ & 0.8 \\
\hline 2020 & 0.2 & -0.5 & 0 & 0.5 & -0.5 & -0.8 & 0.2 & 1 & -0.5 & -0.1 & 0.9 & -0.5 \\
\hline 2021 & $-1.1 *$ & -0.5 & 0 & $-1.2 *$ & 0.3 & 1 & 0.1 & $-1.2 *$ & 0.2 & 0.4 & $-1 *$ & 0.8 \\
\hline 2022 & 2 & -0.5 & 0 & $-1.8 * *$ & -0.9 & 0.4 & 1.7 & -0.6 & -0.2 & 1.2 & 0.2 & 2.3 \\
\hline 2023 & 0.2 & -0.5 & 0 & 0.5 & -0.3 & -0.9 & 1.1 & 0.9 & -0.5 & -0.2 & 0.7 & -0.4 \\
\hline 2024 & $-1.1 *$ & -0.5 & 0 & $-1.2 *$ & 0.3 & 1 & 0.5 & $-1.2 *$ & 0.2 & 0.4 & -0.9 & 0.8 \\
\hline 2025 & -0.2 & -0.5 & 0 & 0.5 & -0.5 & -0.8 & 0.4 & 1 & -0.5 & -0.1 & 0.8 & -0.5 \\
\hline 2026 & 0.2 & -0.5 & 0 & 0.5 & -0.4 & $-1^{*}$ & 0.4 & 0.8 & -0.5 & -0.1 & 0.7 & -0.6 \\
\hline
\end{tabular}


Table 23. Calculated SPI values for Station No. 6 (Ishurdi) (2012-2026)

\begin{tabular}{|c|c|c|c|c|c|c|c|c|c|c|c|c|}
\hline Year & Jan & Feb & Mar & Apr & May & June & July & Aug & Sep & Oct & Nov & Dec \\
\hline $\mathbf{2 0 1 2}$ & 0.1 & -0.6 & 0.9 & 3.6 & -0.1 & -0.9 & -0.1 & 0.9 & -0.6 & 1.7 & 1.3 & 0.1 \\
\hline $\mathbf{2 0 1 3}$ & 0.1 & -0.8 & 0.9 & -0.3 & -0.1 & -0.6 & 0.1 & 0.8 & -0.4 & -0.1 & 0.2 & -0.3 \\
\hline $\mathbf{2 0 1 4}$ & $-1.3^{*}$ & 1.4 & -0.4 & -0.3 & 0.0 & 1.1 & -0.1 & $-1.4^{*}$ & 0.3 & 0.4 & -0.8 & 1.3 \\
\hline $\mathbf{2 0 1 5}$ & 0.1 & $-1^{*}$ & 0.7 & -0.3 & -0.1 & $-1^{*}$ & 0.1 & 0.9 & -0.5 & 0 & 0.6 & -0.4 \\
\hline $\mathbf{2 0 1 6}$ & $-0.4^{*}$ & 1.4 & $-2.8^{* * *}$ & -0.3 & 0.7 & 1.4 & $-2.9^{* * *}$ & -0.6 & 3.3 & $-3.2^{* * *}$ & $-2.6^{* * *}$ & -0.9 \\
\hline $\mathbf{2 0 1 7}$ & 0.1 & $-1^{*}$ & -0.5 & -0.3 & 0 & $-1.1^{*}$ & -0.8 & 0.8 & $-1.1^{*}$ & 0.4 & 1 & -0.2 \\
\hline $\mathbf{2 0 1 8}$ & $-1.3^{*}$ & 1.4 & -0.7 & -0.3 & 0.7 & 1.2 & -0.4 & $-1.4^{*}$ & 0.4 & 0.3 & -0.9 & 1.3 \\
\hline $\mathbf{2 0 1 9}$ & -0.4 & 0.9 & 0.3 & -0.3 & 0.2 & 0.7 & -0.2 & $-1.4 *$ & 0.1 & 0.3 & -0.5 & 1.1 \\
\hline $\mathbf{2 0 2 0}$ & 0.1 & -0.9 & 0.8 & -0.3 & -0.1 & -0.9 & 0.2 & 0.9 & -0.5 & 0.0 & 0.6 & -0.3 \\
\hline $\mathbf{2 0 2 1}$ & 2.3 & -0.1 & -0.4 & -0.3 & -0.2 & 0.1 & 1.4 & -0.1 & -0.1 & 0.3 & 0.9 & -0.2 \\
\hline $\mathbf{2 0 2 2}$ & 0.1 & -0.8 & 0.7 & -0.3 & -0.1 & -0.8 & 0.5 & 0.8 & -0.5 & 0 & 0.3 & -0.2 \\
\hline $\mathbf{2 0 2 3}$ & 1.9 & -0.1 & -0.5 & -0.3 & -0.2 & 1.4 & 1.5 & -0.6 & 0.3 & -0.5 & -0.3 & $-2.5 * * *$ \\
\hline $\mathbf{2 0 2 4}$ & -0.4 & -0.2 & 0.7 & -0.3 & -0.1 & $-1^{*}$ & 0.5 & 0.8 & -0.5 & 0.1 & 0.6 & 0.0 \\
\hline $\mathbf{2 0 2 5}$ & 0.1 & -0.8 & 0.9 & -0.3 & -0.1 & -0.6 & 0.3 & 0.9 & -0.4 & 0.0 & 0.4 & -0.2 \\
\hline $\mathbf{2 0 2 6}$ & $-1.3 *$ & 1.4 & -0.4 & -0.3 & 0 & 1.1 & -0.1 & $-1.4 *$ & 0.3 & 0.3 & -0.8 & 1.3 \\
\hline
\end{tabular}

* Moderate drought, ** Severe drought, *** Extreme drought

\section{Conclusions}

The droughts in the northern region of Bangladesh over the study period varied from moderately dry to severely dry conditions in most years. In future, normally it may vary from moderately dry to severely dry conditions but sometimes extremely dry condition may also occur according to the calculated SPI values. From the study it is observed that the Dinajpur region had faced moderate drought in 1992, 1996, 1997, 1998, 2001, 2006, 2009, 2010; severe drought in 1995, 1992; and this region may face moderate drought in 2013, 2014, 2015, 2021; severe drought in 2015, 2022, 2023, 2024, 2025, 2026; and extreme drought in 2016. The Rangpur region had experienced moderate drought in 1992, 1993, 1994, 1995, 1997, 2000, 2003, 2004, 2007, 2008, 2011; severe drought in 1991, 2000, 2009; and this region may face moderate drought in 2012, 2013, 2014, 2015, 2016, 2017, 2018, 2019, 2020, 2021, 2022, 2023, 2024; severe drought in 2012, 2025, 2026. The Rajshahi region had faced moderate drought in 1992, 1995, 1997, 1998, 1999, 2000, 2003, 2006, 2008, 2010; severe drought in 1991, 1992, 1997, 1998, 2005; and this region may face moderate drought in 2012, 2013, 2014, 2016, 2020, 2022, 2023, 2026; severe drought in 2016, 2021; extreme drought in 2024. The Bogra region had experienced moderate drought in 1992, 1994, 1995, 1997, 1999, 2001, 2002, 2005, 2007, 2009, 2011; severe drought in 2010; and this region may face moderate drought in 2013, 2014, 2015, 2016, 2017, 2018, 2021, 2023, 2024, 2025; severe drought in 2012, 2018, 2019, 2022. The Sydpur region had experience moderate drought in 1991, 1992, 1994, 1995, 2001, 2005, 2006, 2008, 2009, 2011; severe drought in 1991, 1995, 2000; and this region may face moderate drought in 2012, 2014, 2016, 2017, 2019, 2021, 2024; severe drought in 2022 and extreme drought in 2014. The Ishurdi region had experienced moderate drought in 1992, 1994, 1995, 1996, 1999, 2001, 2003, 2005, 2006, 2010; severe drought in 1992, 1994, 1998, 2003, 2007, 2009, 2010; and this region may face moderate drought in 2014, 2015, 2017, 2019, 2024, 2026 and extreme drought in 2016.

\section{References}

Adnan, S. 1993. Living without Floods: Lessons from the Drought of 1992. Dhaka: Research and Advisory Services 1.

Beran, M. A. and Rodier, J. A. 1985. Hydrological Aspects of Drought: a Contribution to the 
International Hydrological Programme. World Meteorological Organization, Studies and reports on hydrology, UNESCO-WMO, Paris, France. 39 Pages.

Daniel, O. 2008. Drought Analysis for Busia District (Uganda). Master in Problematica dellôrrigazione nei Paesi in via di svilu ppo. 127 Pages.

Erickson, N. J.; Ahmad, Q. K. and Chowdhury, A. R. 1993. Socio- Economic Implications of Climate Change for Bangladesh. Dhaka: Bangladesh Unnayan Parishad.

Hossain, M. 1990. Natural Calamities, Instability in Production and Food Policy in Bangladesh. The Bangladesh Development Studies, 18(1): 33-54.
Odongkara, B. 2002. Drought Duration and Frequency Analysis: A Case Study of Northern and Western Uganda. Unpublished Project Report, Department of Civil Engineering, Markerere University Kampala, Uganda.

Rahman, A. and Biswas, P. R. 1995. Devours Resources. Dhaka Courier, 11 (42): 7-8.

Rahman, M. H. 1995. Responding to Drought in Bangladesh. The Daily Star, May 15. 8 Pages.

Smakhtin and Hughes. 2004. Review, automated estimation and analyses of drought indices, in South Asia. Working Paper 83, International Water Management Institute, Colombo, Sri Lanka. 